\title{
Generation and dynamics of entangled fermion-photon-phonon states in nanocavities
}

\author{
Mikhail Tokman, ${ }^{1}$ Maria Erukhimova, ${ }^{1}$ Yongrui \\ Wang, ${ }^{2}$ Qianfan Chen, ${ }^{2}$ and Alexey Belyanin ${ }^{2}$ \\ ${ }^{1}$ Institute of Applied Physics, Russian Academy \\ of Sciences, Nizhny Novgorod, 603950, Russia \\ ${ }^{2}$ Department of Physics and Astronomy, \\ Texas A\&BM University, College Station, TX, 77843 USA
}

(Dated: July 7, 2020)

\begin{abstract}
We develop the analytic theory describing the formation and evolution of entangled quantum states for a fermionic quantum emitter coupled to a quantized electromagnetic field in a nanocavity and quantized phonon or mechanical vibrational modes. The theory is applicable to a broad range of cavity quantum optomechanics problems and emerging research on plasmonic nanocavities coupled to single molecules and other quantum emitters. The optimal conditions for a tri-state entanglement are realized near the parametric resonances in a coupled system. The model includes decoherence effects due to coupling of the fermion, photon, and phonon subsystems to their dissipative reservoirs within the stochastic evolution approach, which is derived from the Heisenberg-Langevin formalism. Our theory provides analytic expressions for the time evolution of the quantum state and observables, and the emission spectra. The limit of a classical acoustic pumping and the interplay between parametric and standard one-photon resonances are analyzed.
\end{abstract}




\section{INTRODUCTION}

There is a lot of recent interest in the quantum dynamics of fermion systems coupled to an electromagnetic (EM) mode in a cavity and quantum or classical mechanical/acoustic oscillations or phonon vibrations. This problem is related to the burgeoning fields of cavity optomechanics [1-3] and quantum acoustics [4 6]. Another example where this situation can be realized is a molecule placed in a plasmonic nanocavity [7, 8]. In this case the fermion system may comprise two or more electron states forming an optical transition, whereas the phonon field is simply a vibrational mode of a molecule. One can also imagine a situation where a quantum emitter such as a quantum dot or an optically active defect in a solid matrix is coupled to the quantized phonon modes of a crystal lattice, which would be an extension of an extremely active field of research on phonon-polaritons or plasmon-phonon-polaritons [9, 10] into a fully quantum regime.

Apart from the fundamental interest, the studies of such systems are motivated by quantum information applications. Indeed, the presence of a classical or quantized acoustic mode provides an extra handle to control the quantum state of a coupled fermion-boson quantum system. In the extreme quantum limit in which the fermionic degree of freedom and all bosonic degrees of freedom (both photons and phonons) are quantized, a strong enough coupling between them leads to an entangled fermion-photon-phonon state, which is a complex enough system to implement basic gates for quantum computation or other applications. Such a system has not been realized experimentally. However, many ingredients have been already demonstrated, such as strong coupling between a nanocavity mode and a single molecule [11, numerous examples of strong coupling between nanocavity modes and a sin-

gle fermionic quantum emitter such as a color center [12] or a quantum dot (QD) (see e.g. Refs. [13, 14] for semiconductor cavity-QD systems and Refs. [15-17] for plasmonic cavities), strong coupling and entanglement of acoustic phonons [18, 19], resolving the energy levels of a nanomechanical oscillator [6], or cooling a macroscopic system into its motional ground state [20].

Interaction of three or more modes of oscillations, whether they are classical or quantized, is strongly enhanced close to the parametric resonance, which is therefore the most interesting region to study. Fortunately for theorists, the analysis near the parametric resonance is greatly simplified, because some form of a slowly varying amplitude method for 
classical systems [21, 22] or the rotating wave approximation (RWA) for quantum systems 23] can be applied. The use of RWA restricts the coupling strength to the values much lower than the characteristic energies in the system, such as the optical transition or vibrational energy. The emerging studies of the so-called ultra-strong coupling regime [24] have to go beyond the RWA. Nevertheless, for the vast majority of experiments, including nonperturbative strong coupling dynamics and entanglement, the RWA is adequate and provides some crucial simplifications that allow one to obtain analytic solutions.

In particular, within Schrödinger's description, the equations of motion for the components of an infinitely dimensional state vector $|\Psi\rangle$ that describes a coupled fermion-boson system can be split into the blocks of low dimensions if the RWA is applied. This is true even if the dynamics of the fermion subsystem is nonperturbative, e.g. the effects of saturation are important. Note that there is no such simplification in the Heisenberg representation, i.e. when solving the equations of motion

$$
\frac{d}{d t} \hat{g}=\frac{i}{\hbar}[\hat{H}, \hat{g}],
$$

where $\widehat{g}$ is the Heisenberg operator of a certain physical observable $g$ and $\hat{H}$ is the Hamiltonian of the system. Operator-valued Eqs. (1) are generally impossible to split into smaller blocks, even within the RWA. This happens because some matrix elements $g_{A B}(t)$ of the Heisenberg operator are determined by states $|A\rangle,|B\rangle$ which belong to different blocks that evolve independently in the Schrödinger picture. The simplification could only be possible for specially selected initial conditions in which the Heisenberg operator is determined on a "truncated" basis belonging to only one of the independent blocks. The Schrödinger's approach also leads to fewer equations for the state vector components than the approach based on the von Neumann master equation for the elements of the density matrix.

Obviously, the Schrödinger equation in its standard form cannot be applied to describe open systems coupled to a dissipative reservoir. In this case the stochastic versions of the equation of evolution for the state vector have been developed, e.g. the method of quantum jumps [23, 25]. This method is optimal for numerical analysis in the Monte-Carlo type schemes. Here we formulate the stochastic equation for the state vector derived from the Heisenberg-Langevin approach which is more conducive to the analytic treatment. Its key element is an assumption that there exists the operator of evolution $\hat{U}$, which is determined unambiguously not only by the parameters of the dynamical system but also by the statistical 
properties of a dissipative reservoir.

The paper is structured as follows. Section II formulates the model and the Hamiltonian for coupled quantized fermion, photon, and phonon fields in a nanocavity. Section III derives the solution for the quantum states of a closed system in the vicinity of a parametric resonance and analyze its properties. In Section IV we provide the stochastic equation describing the evolution of quantum states of an open system in contact with a dissipative reservoir and describe the observables. In Section $\mathrm{V}$ we consider the case of a classical acoustic pumping. Section VI describes the interplay of parametric and standard one-photon resonances and provides the conditions under which these resonances can be separated. Section VII gives an example of manipulating entangled electron-photon states by an acoustic pumping. Appendix contains the derivation of the stochastic equation of evolution from the Heisenberg-Langevin approach and compares with Lindblad density-matrix formalism.

\section{A COUPLED QUANTIZED ELECTRON-PHOTON-PHONON SYSTEM: THE MODEL}

Consider a quantized electron system coupled to the quantum EM field of a nanocavity and classical or quantized vibrational (phonon) modes, see Fig. 1 which sketches two out of many possible scenarios.

Here the electron transition energy is $W$, the photon and phonon mode frequencies are $\omega$ and $\Omega$, respectively. The decay constants $\gamma, \mu_{\omega}$, and $\mu_{\Omega}$ of the electron, photon, and phonon subsystems due to couplings to their respective dissipative reservoirs are also indicated. Figure 1b implies that it is a quantum dot which experiences vibrations, but our treatment below works for any mechanism of relative displacement between the electron system and the field of an EM cavity mode, including the situations where it is the wall of a nanocavity which experiences oscillations.

We start from writing down a general Hamiltonian for a coupled quantized electronphoton-phonon system and derive its various approximate forms: the RWA, small-amplitude acoustic oscillations, classical vs. quantum phonon mode, etc. 


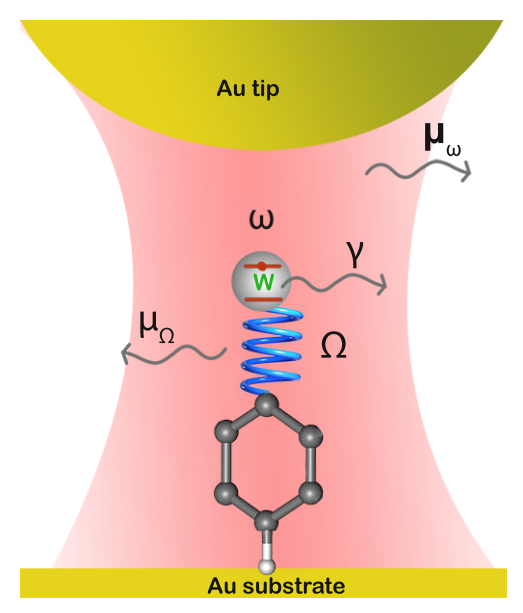

(a)

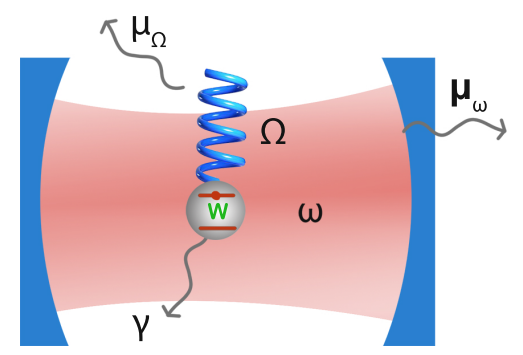

(b)

FIG. 1: (a) A sketch of a molecule in a nanocavity created by a metallic nanotip and a substrate; (b) A sketch of a quantum dot coupled to optical and mechanical vibrational modes in a nanocavity.

\section{A. The fermion subsystem}

Consider the simplest version of the fermion subsystem: two electron states $|0\rangle$ and $|1\rangle$ with energies 0 and $W$, respectively. We will call it an "atom" for brevity, although it can be electron states of a molecule, a quantum dot, or any other electron system. Introduce creation and annihilation operators of the excited state $|1\rangle, \hat{\sigma}=|0\rangle\left\langle 1\left|, \hat{\sigma}^{\dagger}=\right| 1\right\rangle\langle 0|$, which satisfy standard commutation relations for fermions:

$$
\hat{\sigma}^{\dagger}|0\rangle=|1\rangle, \hat{\sigma}|1\rangle=|0\rangle, \hat{\sigma} \hat{\sigma}=\hat{\sigma}^{\dagger} \hat{\sigma}^{\dagger}=0 ;\left[\hat{\sigma}, \hat{\sigma}^{\dagger}\right]_{+}=\hat{\sigma} \hat{\sigma}^{\dagger}+\hat{\sigma}^{\dagger} \hat{\sigma}=1
$$

The Hamiltonian of an atom is

$$
\hat{H}_{a}=W \hat{\sigma}^{\dagger} \hat{\sigma}
$$


We will also need the dipole moment operator,

$$
\hat{\mathbf{d}}=\mathbf{d}\left(\hat{\sigma}^{\dagger}+\hat{\sigma}\right)
$$

where $\mathbf{d}=\langle 1|\hat{\mathbf{d}}| 0\rangle$ is a real vector. For a finite motion we can always choose the coordinate representation of stationary states in terms of real functions.

\section{B. Quantized EM modes of a cavity}

We use a standard representation for the electric field operator in a cavity:

$$
\hat{\mathbf{E}}=\sum_{i}\left[\mathbf{E}_{i}(\mathbf{r}) \hat{c}_{i}+\mathbf{E}_{i}^{*}(\mathbf{r}) \hat{c}_{i}^{\dagger}\right]
$$

where $\hat{c}_{i}^{\dagger}, \hat{c}_{i}$ are creation and annihilation operators for photons at frequency $\omega_{i}$; the functions $\mathbf{E}_{i}(\mathbf{r})$ describe the spatial structure of the EM modes in a cavity. The relation between the modal frequency $\omega_{i}$ and the function $\mathbf{E}_{i}(\mathbf{r})$ can be found by solving the boundary-value problem of the classical electrodynamics [23]. The normalization conditions [26]

$$
\int_{V} \frac{\partial\left[\omega_{i}^{2} \varepsilon\left(\omega_{i}, \mathbf{r}\right)\right]}{\omega_{i} \partial \omega_{i}} \mathbf{E}_{i}^{*}(\mathbf{r}) \mathbf{E}_{i}(\mathbf{r}) d^{3} r=4 \pi \hbar \omega_{i}
$$

ensure correct bosonic commutators $\left[\hat{c}_{i}, \hat{c}_{i}^{\dagger}\right]=\delta_{i j}$ and the field Hamiltonian in the form

$$
\hat{H}_{e m}=\hbar \sum_{i} \omega_{i}\left(\hat{c}_{i}^{\dagger} \hat{c}_{i}+\frac{1}{2}\right) .
$$

Here $V$ is a quantization volume and $\varepsilon(\omega, \mathbf{r})$ is the dielectric function of a dispersive medium that fills the cavity.

\section{The quantized phonon field}

We assume that our two-level atom is dressed by a phonon field which can be described by the displacement operator:

$$
\hat{\mathbf{q}}=\sum_{i} \hat{\mathbf{q}}_{i} ; \quad \hat{\mathbf{q}}_{i}=\mathbf{Q}_{i}(\mathbf{r}) \hat{b}_{i}+\mathbf{Q}_{i}^{*}(\mathbf{r}) \hat{b}_{i}^{\dagger}
$$

Here $\hat{b}_{i}$ and $\hat{b}_{i}^{\dagger}$ are annihilation and creation operators of phonons and the functions $\mathbf{Q}_{i}(\mathbf{r})$ determine the spatial structure of oscillations at frequencies $\Omega_{i}$. Expression (7) can be used 
when the amplitude of oscillations is small enough. One can always choose the normalization

of functions $\mathbf{Q}_{i}(\mathbf{r})$ corresponding to standard commutation relations for bosons, $\left[\hat{b}_{i}, \hat{b}_{j}^{\dagger}\right]=\delta_{i j}$ and a standard form for the Hamiltonian of mechanical oscillations:

$$
\hat{H}_{p}=\hbar \sum_{i} \Omega_{i}\left(\hat{b}_{i}^{\dagger} \hat{b}_{i}+\frac{1}{2}\right) .
$$

\section{An atom coupled to quantized EM and phonon fields}

Now we can combine all ingredients into a coupled quantized system. Adding the interaction Hamiltonian with a EM cavity mode in the electric dipole approximation, $-\hat{\mathbf{d}} \cdot \hat{\mathbf{E}}$, the Hamiltonian of an atom coupled to a single mode EM field

$$
\hat{H}=\hat{H}_{e m}+\hat{H}_{a}-\mathbf{d}\left(\hat{\sigma}^{\dagger}+\hat{\sigma}\right) \cdot\left[\mathbf{E}(\mathbf{r}) \hat{c}+\mathbf{E}^{*}(\mathbf{r}) \hat{c}^{\dagger}\right]_{\mathbf{r}=\mathbf{r}_{a}}
$$

where $\mathbf{r}=\mathbf{r}_{a}$ denotes the position of an atom inside the cavity. The effect of "dressing" of the coupled atom-EM field system by mechanical oscillations in its most general form can be included by adding the Hamiltonian of phonon modes $\hat{H}_{p}$ and substituting $\mathbf{r}_{a} \Longrightarrow \mathbf{r}_{a}+\hat{\mathbf{q}}$ in Eq. (9). This will work for an arbitrary relative displacement of an atom with respect to the EM cavity mode. Keeping only one phonon mode for simplicity, in which

$$
\hat{\mathbf{q}}=\mathbf{Q}(\mathbf{r}) \hat{b}+\mathbf{Q}^{*}(\mathbf{r}) \hat{b}^{\dagger}
$$

and expanding in Taylor series in the vicinity of $\mathbf{r}=\mathbf{r}_{a}$, we obtain the total Hamiltonian,

$$
\begin{aligned}
\hat{H}= & \hat{H}_{e m}+\hat{H}_{a}+\hat{H}_{p}-\left(\chi \hat{\sigma}^{\dagger} \hat{c}+\chi^{*} \hat{\sigma} \hat{c}^{\dagger}+\chi \hat{\sigma} \hat{c}+\chi^{*} \hat{\sigma}^{\dagger} \hat{c}^{\dagger}\right) \\
& -\left(\eta_{1} \hat{\sigma}^{\dagger} \hat{c} \hat{b}+\eta_{1}^{*} \hat{\sigma} \hat{c}^{\dagger} \hat{b}^{\dagger}+\eta_{2} \hat{\sigma}^{\dagger} \hat{c} \hat{b}^{\dagger}+\eta_{2}^{*} \hat{\sigma} \hat{c}^{\dagger} \hat{b}+\eta_{1} \hat{\sigma} \hat{c} \hat{b}+\eta_{1}^{*} \hat{\sigma}^{\dagger} \hat{c}^{\dagger} \hat{b}^{\dagger}+\eta_{2} \hat{\sigma} \hat{c} \hat{b}^{\dagger}+\eta_{2}^{*} \hat{\sigma}^{\dagger} \hat{c}^{\dagger} \hat{b}\right)(11
\end{aligned}
$$

where

$$
\chi=(\hat{\mathbf{d}} \cdot \hat{\mathbf{E}})_{\mathbf{r}=\mathbf{r}_{a}}, \eta_{1}=[\mathbf{d}(\mathbf{Q} \cdot \nabla) \mathbf{E}]_{\mathbf{r}=\mathbf{r}_{a}}, \eta_{2}=\left[\mathbf{d}\left(\mathbf{Q}^{*} \cdot \nabla\right) \mathbf{E}\right]_{\mathbf{r}=\mathbf{r}_{a}} .
$$

Note that we can always take the functions $\mathbf{E}(\mathbf{r})$ and $\mathbf{Q}(\mathbf{r})$ to be real at the position of an atom, but we cannot keep the derivatives real at the same time if the modal structure $\propto e^{i \mathbf{k} \cdot \mathbf{r}}$. However, for ideal cavity modes the latter is possible. As we will see below, the best conditions for electron-photon-phonon entanglement are reached in the vicinity of the parametric resonance:

$$
\frac{W}{\hbar} \approx \omega \pm \Omega
$$


When the upper sign is chosen in Eq. (12), the RWA applied to the Hamiltonian (11) yields

$$
\hat{H}=\hat{H}_{e m}+\hat{H}_{a}+\hat{H}_{p}-\left(\eta \hat{\sigma}^{\dagger} \hat{c} \hat{b}+\eta^{*} \hat{\sigma} \hat{c}^{\dagger} \hat{b}^{\dagger}\right)
$$

where $\eta \equiv \eta_{1}$. For the lower sign in Eq. (12), the RWA Hamiltonian is

$$
\hat{H}=\hat{H}_{e m}+\hat{H}_{a}+\hat{H}_{p}-\left(\eta \hat{\sigma}^{\dagger} \hat{c} \hat{b}^{\dagger}+\eta^{*} \hat{\sigma} \hat{c}^{\dagger} \hat{b}\right)
$$

where $\eta \equiv \eta_{2}$.

\section{E. An atom coupled to the quantized EM field and dressed by a classical acoustic} field

For classical acoustic oscillations the operator $\hat{\mathbf{q}}=\mathbf{Q}(\mathbf{r}) \hat{b}+\mathbf{Q}^{*}(\mathbf{r}) \hat{b}^{\dagger}$ in Eq. 10 becomes a classical function

$$
\mathbf{q}=\mathbf{Q}(\mathbf{r}) e^{-i \Omega t}+\mathbf{Q}^{*}(\mathbf{r}) e^{i \Omega t}
$$

where $\mathbf{Q}$ is a coordinate-dependent complex amplitude of classical oscillations. Near the parametric resonance $\left(\omega+\Omega \approx \frac{W}{\hbar}\right)$ the RWA Hamiltonian takes the form

$$
\hat{H}=\hat{H}_{e m}+\hat{H}_{a}-\left(\mathfrak{R} \hat{\sigma}^{\dagger} \hat{c} e^{-i \Omega t}+\mathfrak{R}^{*} \hat{\sigma} \hat{c}^{\dagger} e^{i \Omega t}\right) .
$$

where $\mathfrak{R}=[\mathbf{d}(\mathbf{Q} \cdot \nabla) \mathbf{E}]_{\mathbf{r}=\mathbf{r}_{a}}$. The value of the acoustic frequency $\Omega$ in Eq. (16) can be of either sign, corresponding to the choice " \pm " in the parametric resonance condition Eq. (12); When the sign of $\Omega$ changes from positive to negative, one should replace $\mathbf{Q}$ with $\mathbf{Q}^{*}$ in the above expression for $\mathfrak{R}$.

Qualitatively, Hamiltonian (13) corresponds to the decay of the fermionic excitation into a photon and phonon; Hamiltonian (14) corresponds to the decay of a photon into a phonon and fermionic excitation, whereas Hamiltonian 16 describes parametric decay of a photon into an atomic excitation and back, mediated by classical acoustic oscillations.

\section{PARAMETRIC RESONANCE IN A CLOSED SYSTEM}

When the system is closed and there is no dissipation, the general analytic solution to the dynamics of coupled fermions, photons, and phonons can be obtained in the RWA. We write the state vector as 


$$
\Psi=\sum_{\alpha, n=0}^{\infty}\left(C_{\alpha n 0}|\alpha\rangle|n\rangle|0\rangle+C_{\alpha n 1}|\alpha\rangle|n\rangle|1\rangle\right) .
$$

Here Greek letters denote phonon states, Latin letters denote photon states, and numbers 0, 1 describe fermion states. We will keep the same sequence of symbols throughout the paper:

$$
\left.\left.\left.C_{\text {phonon photon fermion }} \mid \text { phonon }\right\rangle \mid \text { photon }\right\rangle \mid \text { fermion }\right\rangle \text {. }
$$

Next, we substitute Eq. (17) into the Schrödinger equation,

$$
i \hbar \frac{\partial}{\partial t}|\Psi\rangle=\hat{H}|\Psi\rangle
$$

Where $\hat{H}$ is the RWA Hamiltonian. For definiteness, we consider the vicinity of the parametric resonance with a plus sign, $\omega+\Omega \approx \frac{W}{\hbar}$, which corresponds to the Hamiltonian $(13)$. In this case the equations for the coefficients in Eq. (17) can be separated into the pairs of coupled equations

$$
\frac{d}{d t}\left(\begin{array}{c}
C_{\alpha n 0} \\
C_{(\alpha-1)(n-1) 1}
\end{array}\right)+\left(\begin{array}{cc}
i \omega_{\alpha, n} & -i \Omega_{R}^{(\alpha, n) *} \\
-i \Omega_{R}^{(\alpha, n)} & i \omega_{\alpha, n}-i \Delta
\end{array}\right)\left(\begin{array}{c}
C_{\alpha n 0} \\
C_{(\alpha-1)(n-1) 1}
\end{array}\right)=0
$$

and a separate equation for the lowest-energy state:

$$
\dot{C}_{000}+i \omega_{0,0} C_{000}=0
$$

where

$$
\Omega_{R}^{(\alpha, n)}=\frac{\eta}{\hbar} \sqrt{\alpha n}, \omega_{\alpha, n}=\Omega\left(\alpha+\frac{1}{2}\right)+\omega\left(n+\frac{1}{2}\right), \Delta=\Omega+\omega-\frac{W}{\hbar} .
$$

Note that approximate Eqs. (19), 20 preserve the norm exactly:

$$
\left|C_{000}\right|^{2}+\sum_{\alpha=1, n=1}^{\infty, \infty}\left(\left|C_{\alpha n 0}\right|^{2}+\left|C_{(\alpha-1)(n-1) 1}\right|^{2}\right)=\sum_{\alpha=0, n=0}^{\infty, \infty}\left(\left|C_{\alpha n 0}\right|^{2}+\left|C_{\alpha n 1}\right|^{2}\right)=\text { const. }
$$

The solution to Eq. (20) is trivial: $C_{000}(t)=C_{000}(0) \exp \left(-i \omega_{0,0} t\right)$. The solution to Eqs. (19) takes the form

$$
\left(\begin{array}{c}
C_{\alpha n 0} \\
C_{(\alpha-1)(n-1) 1}
\end{array}\right)=A e^{-\Lambda_{1}^{(\alpha, n)} t}\left(\begin{array}{c}
1 \\
a_{1}^{(\alpha, n)}
\end{array}\right)+B e^{-\Lambda_{2}^{(\alpha, n)} t}\left(\begin{array}{c}
1 \\
a_{2}^{(\alpha, n)}
\end{array}\right)
$$


where the constants $A$ and $B$ are determined from initial conditions. Here the eigenvalues $\Lambda_{1,2}^{(\alpha, n)}$ and eigenvectors $\left(\begin{array}{c}1 \\ a_{1,2}^{(\alpha, n)}\end{array}\right)$ of the matrix of coefficients in Eq. 190 are given by

$$
\Lambda_{1,2}^{(\alpha, n)}=i \omega_{\alpha, n}-i \delta_{1,2}^{(\alpha, n)}, a_{1,2}^{(\alpha, n)}=\frac{\delta_{1,2}^{(\alpha, n)}}{\Omega_{R}^{(\alpha, n) *}},
$$

where

$$
\delta_{1,2}^{(\alpha, n)}=\frac{\Delta}{2} \pm \sqrt{\frac{\Delta^{2}}{4}+\left|\Omega_{R}^{(\alpha, n)}\right|^{2}}
$$

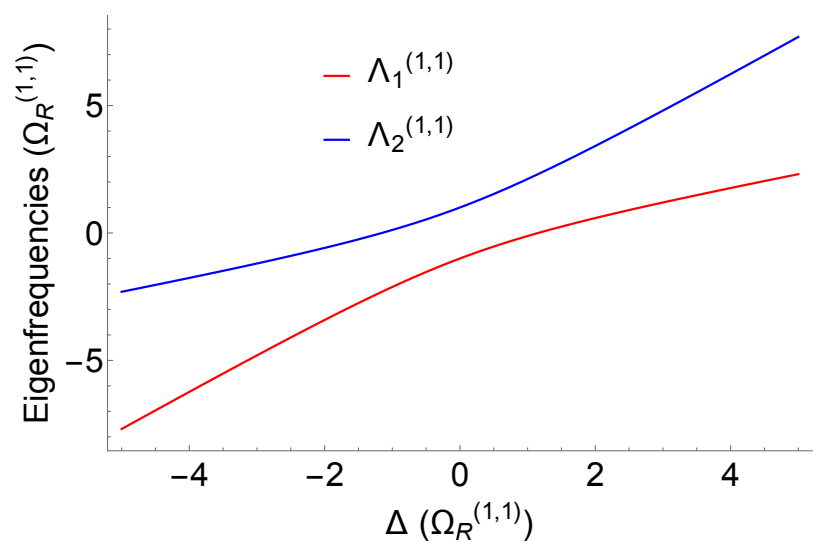

FIG. 2: Frequency eigenvalues of the coupled electron-photon-phonon quantum system as a function of detuning from the parametric resonance $\frac{W}{\hbar}=\Omega+\omega$. All frequencies are in units of the generalized Rabi frequency $\Omega_{R}^{(1,1)}$. The values of eigenfrequencies are shifted vertically by $\left.\omega_{1,1}\right|_{\Delta=0}$.

Fig. 2 shows the eigenfrequencies of the system given by Eq. (22) with $\alpha=n=1$, shifted by $\left.\omega_{1,1}\right|_{\Delta=0}$. One can see the anticrossing with splitting by $2 \Omega_{R}^{(1,1)}$ at the parametric resonance.

As an example, consider an exact parametric resonance, $\frac{W}{\hbar}=\Omega+\omega$ and the simplest initial state $\Psi_{0}=|0\rangle|0\rangle|1\rangle$ corresponding to the initially excited atom in a cavity. In this case the only nonzero amplitudes are $C_{001}$ and $C_{110}$ :

$$
\left(\begin{array}{c}
C_{110} \\
C_{001}
\end{array}\right)=\frac{1}{2} e^{-i\left(\omega_{1,1}-\left|\Omega_{R}^{(1,1)}\right|\right) t}\left(\begin{array}{c}
e^{-i \theta} \\
1
\end{array}\right)+\frac{1}{2} e^{-i\left(\omega_{1,1}+\left|\Omega_{R}^{(1,1)}\right|\right) t}\left(\begin{array}{c}
-e^{-i \theta} \\
1
\end{array}\right),
$$

where

$$
\omega_{1,1}=\Omega\left(1+\frac{1}{2}\right)+\omega\left(1+\frac{1}{2}\right), \Omega_{R}^{(1,1)}=\frac{\eta}{\hbar}=\left|\Omega_{R}^{(1,1)}\right| e^{i \theta}
$$


The resulting state vector is

$$
\Psi=e^{-i \omega_{1,1} t}\left[i e^{-i \theta} \sin \left(\left|\Omega_{R}^{(1,1)}\right| t\right)|1\rangle|1\rangle|0\rangle+\cos \left(\left|\Omega_{R}^{(1,1)}\right| t\right)|0\rangle|0\rangle|1\rangle\right]
$$

This is clearly an entangled electron-photon-phonon state, which is not surprising. In the absence of dissipation, any coupling between these subsystems leads to entanglement.

The dynamics of the corresponding physical observables, such as the energy of the field and the atom, is Rabi oscillations at the frequency which generalizes a standard Rabi frequency to the case of a parametric photon-phonon-atom resonance and which depends on both the spatial structure of the photon and phonon fields and their occupation numbers:

$$
\begin{gathered}
\left\langle\Psi\left|\hat{\mathbf{E}}^{2}\right| \Psi\right\rangle=|\mathbf{E}(\mathbf{r})|^{2}\left[2-\cos \left(2\left|\Omega_{R}^{(1,1)}\right| t\right)\right] \\
\left\langle\Psi\left|\hat{H}_{a}\right| \Psi\right\rangle=W \frac{1+\cos \left(2\left|\Omega_{R}^{(1,1)}\right| t\right)}{2}
\end{gathered}
$$

It is illustrated in Fig. 3 which shows the normalized EM field energy density and energy of an atom as a function of time. Note that the EM field energy never reaches zero because of the presence of zero-point vacuum energy. With detuning from the parametric resonance, the amplitude of the oscillations will decrease.

\section{DYNAMICS OF AN OPEN ELECTRON-PHOTON-PHONON SYSTEM}

\section{A. Stochastic evolution equation}

Now we include the processes of relaxation and decoherence in an open system, which is (weakly) coupled to a dissipative reservoir. We will use the stochastic equation of evolution for the state vector, which is derived in Appendix. This is basically the Schrödinger equation modified by adding a linear relaxation operator and the noise source term with appropriate correlation properties. The latter are related to the parameters of the relaxation operator, which is a manifestation of the fluctuation-dissipation theorem [31]. In Appendix we derived the general form of the stochastic equation of evolution from the Heisenberg-Langevin equations [23, 27, 28] and showed how physically reasonable constraints on the observables determine the properties of the noise sources. We also demonstrated the relationship between our approach and the Lindblad method of solving the master equation. 


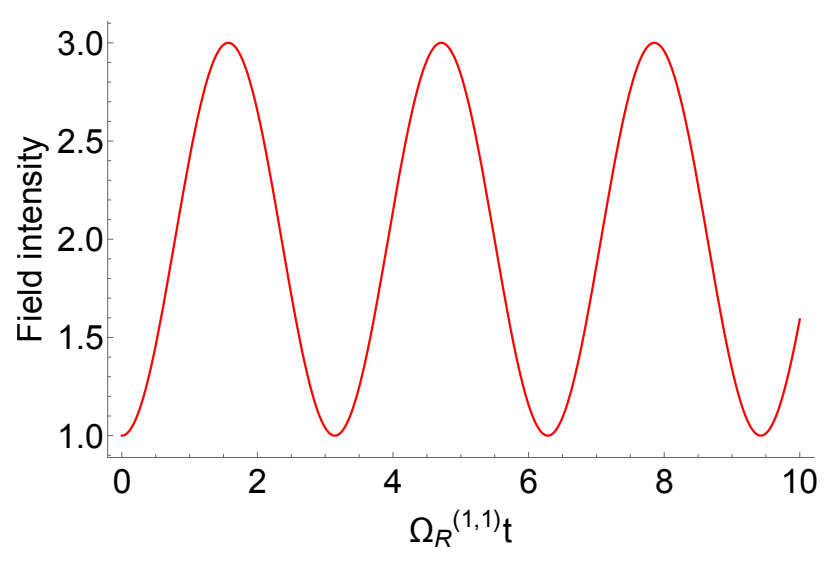

(a)

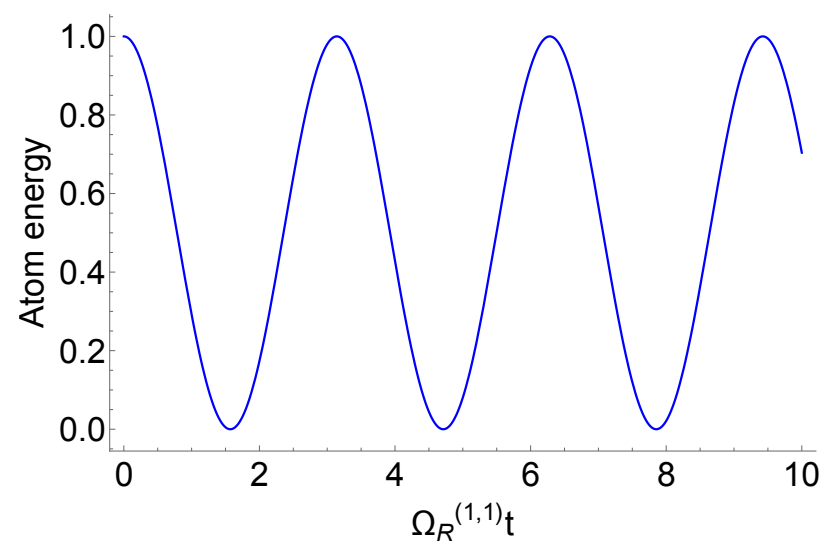

(b)

FIG. 3: (a) Normalized field intensity, $\left\langle\Psi\left|\hat{\mathbf{E}}^{2}\right| \Psi\right\rangle /|\mathbf{E}(\mathbf{r})|^{2}$, and (b) normalized atom energy $\left\langle\Psi\left|\hat{H}_{a}\right| \Psi\right\rangle / W$ as a function of time in units of the generalized Rabi frequency $\Omega_{R}^{(1,1)}$.

Within our approach the system is described by a state vector which has a fluctuating component: $|\Psi\rangle=\overline{|\Psi\rangle}+\widetilde{\Psi \Psi\rangle}$, where the straight bar means averaging over the statistics of noise and the wavy bar denotes the fluctuating component. This state vector is of course very different from the state vector obtained by solving a standard Schrödinger equation for a closed system. In fact, coupling to a dissipative reservoir leads to the formation of a

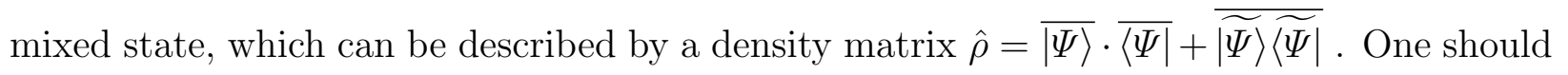
view the stochastic equation approach as a convenient formalism for calculating physical observables.

Following the derivation in Appendix, Eqs. 19, 20 are modified as 


$$
\begin{aligned}
& \frac{d}{d t}\left(\begin{array}{c}
C_{\alpha n 0} \\
C_{(\alpha-1)(n-1) 1}
\end{array}\right)+\left(\begin{array}{cc}
i \omega_{\alpha, n}+\gamma_{\alpha n 0} & -i \Omega_{R}^{(\alpha, n) *} \\
-i \Omega_{R}^{(\alpha, n)} & i \omega_{\alpha, n}-i \Delta+\gamma_{(\alpha-1)(n-1) 1}
\end{array}\right)\left(\begin{array}{c}
C_{\alpha n 0} \\
C_{(\alpha-1)(n-1) 1}
\end{array}\right) \\
&=-\frac{i}{\hbar}\left(\begin{array}{c}
R_{\alpha n 0} \\
R_{(\alpha-1)(n-1) 1}
\end{array}\right), \\
& \dot{C}_{000}+\left(i \omega_{0,0}+\gamma_{000}\right) C_{000}=-\frac{i}{\hbar} R_{000} .
\end{aligned}
$$

Coupling to a reservoir introduces two main differences to Eqs. 28, 29) as compared to Eqs. (19, 20 for a closed system. First, eigenfrequencies acquire imaginary parts which describe relaxation:

$$
\omega_{\alpha, n} \Longrightarrow \omega_{\alpha, n}-i \gamma_{\alpha n 0}, \omega_{\alpha, n}-\Delta \Longrightarrow \omega_{\alpha, n}-\Delta-i \gamma_{(\alpha-1)(n-1) 1}, \omega_{0,0} \Longrightarrow \omega_{0,0}-i \gamma_{000}
$$

The relaxation constants are determined by the properties of all subsystems. They are derived in Appendix and their explicit form is given in the end of this section.

Second, the right-hand side of Eqs. (28) and 29 contain noise sources $-\frac{i}{\hbar} R_{\alpha n 0},-\frac{i}{\hbar} R_{(\alpha-1)(n-1) 1}$ and $-\frac{i}{\hbar} R_{000}$. They are equal to 0 after averaging over the noise statistics: $\overline{R_{\alpha n} 0}=$ $\overline{R_{(\alpha-1)(n-1) 1}}=\overline{R_{000}}$. The averages of the quadratic combinations of noise source terms are nonzero. Including the noise sources is crucial for consistency of the formalism: it ensures the conservation of the norm of the state vector and leads to a physically meaningful equilibrium state. Note that the Weisskopf-Wigner theory does not enforce the conservation of the norm.

\section{B. Evolution of the state amplitudes and observables}

The solution to Eq. (29) is

$$
C_{000}=e^{-\left(i \omega_{0,0}+\gamma_{000}\right) t}\left[C_{000}(0)-\frac{i}{\hbar} \int_{0}^{t} e^{\left(i \omega_{0,0}+\gamma_{000}\right) \tau} R_{000}(\tau) d \tau\right]
$$

The solution to Eqs. 28) is determined again by the eigenvalues and eigenvectors of the matrix of coefficients, which are now modified by relaxation rates:

$$
\Lambda_{1,2}^{(\alpha, n)}=i \omega_{\alpha, n}-i \delta_{1,2}^{(\alpha, n)}, a_{1,2}^{(\alpha, n)}=\frac{\delta_{1,2}^{(\alpha, n)}-i \gamma_{\alpha n 0}}{\Omega_{R}^{(\alpha, n) *}}
$$


where

$$
\delta_{1,2}^{(\alpha, n)}=\frac{\Delta}{2}+i \frac{\gamma_{\alpha n 0}+\gamma_{(\alpha-1)(n-1) 1}}{2} \pm \sqrt{\frac{\left[\Delta+i\left(\gamma_{(\alpha-1)(n-1) 1}-\gamma_{\alpha n 0}\right)\right]^{2}}{4}+\left|\Omega_{R}^{(\alpha, n)}\right|^{2}}
$$

The solution to Eqs. 28) takes the form

$$
\begin{aligned}
& \left(\begin{array}{c}
C_{\alpha n 0} \\
C_{(\alpha-1)(n-1) 1}
\end{array}\right) \\
= & e^{-\Lambda_{1}^{(\alpha, n)} t}\left(\begin{array}{c}
1 \\
a_{1}^{(\alpha, n)}
\end{array}\right)\left(A-\frac{i}{\hbar} \int_{0}^{t} e^{\Lambda_{1}^{(\alpha, n)} \tau} \frac{R_{\alpha n 0}(\tau) a_{2}^{(\alpha, n)}-R_{(\alpha-1)(n-1) 1}(\tau)}{\left.a_{2}^{(\alpha, n)}-a_{1}^{(\alpha, n)} d \tau\right)}\right. \\
+ & e^{-\Lambda_{2}^{(\alpha, n)} t}\left(\begin{array}{c}
1 \\
a_{2}^{(\alpha, n)}
\end{array}\right)\left(B-\frac{i}{\hbar} \int_{0}^{t} e^{\Lambda_{2}^{(\alpha, n)} \tau} \frac{R_{(\alpha-1)(n-1) 1}(\tau)-R_{\alpha n 0}(\tau) a_{1}^{(\alpha, n)}}{a_{2}^{(\alpha, n)}-a_{1}^{(\alpha, n)}} d \tau\right)
\end{aligned}
$$

Where the constants $A$ and $B$ are determined by initial conditions.

As an example, we consider the reservoir at low temperatures, when the steady-state population should go to the ground state $|0\rangle|0\rangle|0\rangle$. In this case we can take $\gamma_{000}=0$, as shown below. We will also assume that the only nonzero correlator of noise is delta-correlated in time:

$$
\overline{R_{000}(t+\xi) R_{000}^{*}(t)}=\hbar^{2} \delta(\xi) D_{000}
$$

Then Eqs. 29 and 30 yield

$$
\frac{d}{d t} \overline{\left|C_{000}\right|^{2}}=D_{000}
$$

whereas Eqs. 28 give

$$
\frac{d}{d t}\left(\overline{\left|C_{\alpha n 0}\right|^{2}}+\overline{\left|C_{(\alpha-1)(n-1) 1}\right|^{2}}\right)=-2\left(\gamma_{\alpha n 0} \overline{\left|C_{\alpha n 0}\right|^{2}}+\gamma_{(\alpha-1)(n-1) 1} \overline{\left|C_{(\alpha-1)(n-1) 1}\right|^{2}}\right) .
$$

This equation guarantees that the system occupies the ground state at $t \rightarrow \infty$.

The noise intensity is determined by the condition that the norm of the state vector be conserved. This gives

$$
D_{000}=2 \sum_{\alpha=1, n=1}^{\infty, \infty}\left(\gamma_{\alpha n 0} \overline{\left|C_{\alpha n 0}\right|^{2}}+\gamma_{(\alpha-1)(n-1) 1} \overline{\left|C_{(\alpha-1)(n-1) 1}\right|^{2}}\right) .
$$

In Appendix we discuss in detail the dependence of the noise correlator on the averaged dyadic components of the state vector. We also show how to find the correlators which ensure that the system approaches thermal distribution at a finite temperature. 
The above formalism allows us to obtain analytic solutions to the state vector and observables at any temperatures and detunings from the parametric resonance, while still within the RWA limits. However, the resulting expressions are very cumbersome and they are better to visualize in the plots. Let's give an example of the solution at zero reservoir temperature and exactly at the parametric resonance $\frac{W}{\hbar}=\Omega+\omega$, when the expressions are more manageable. Consider the initial state $\Psi_{0}=|0\rangle|0\rangle|1\rangle$ when an atom is excited and boson modes are in the ground state. In this case the only nonzero amplitudes are $C_{000}$, $C_{001}$ and $C_{110}$. To make the algebra a bit simpler, we assume that the dissipation is weak enough and its effect on the eigenvectors $\left(\begin{array}{c}1 \\ a_{1,2}^{(\alpha, n)}\end{array}\right)$ can be neglected. As a result, we obtain $\Psi=e^{-\left(i \omega_{1,1}-\frac{\gamma_{001}+\gamma_{110}}{2}\right) t}\left[i e^{-i \theta} \sin \left(\left|\widetilde{\Omega}_{R}^{(1,1)}\right| t\right)|1\rangle|1\rangle|0\rangle+\cos \left(\left|\widetilde{\Omega}_{R}^{(1,1)}\right| t\right)|0\rangle|0\rangle|1\rangle\right]+C_{000}|0\rangle|0\rangle|0\rangle$,

where

$$
\overline{\left|C_{000}\right|^{2}}=1-e^{-\left(\gamma_{110}+\gamma_{001}\right) t}, \widetilde{\Omega}_{R}^{(1,1)}=\sqrt{\left|\Omega_{R}^{(1,1)}\right|^{2}-\frac{\left(\gamma_{001}-\gamma_{110}\right)^{2}}{4}}, \theta=\operatorname{Arg}\left[\Omega_{R}^{(1,1)}\right] .
$$

As we see, dissipation leads not only to the relaxation of the entangled part of the state vector, but also to the frequency shift of the Rabi oscillations. This shift is absent if $\gamma_{001}=$ $\gamma_{110}$

The resulting expressions for the observables, such as the EM field intensity and the energy of the atomic excitation are

$$
\begin{gathered}
\left\langle\Psi\left|\hat{\mathbf{E}}^{2}\right| \Psi\right\rangle=|\mathbf{E}(\mathbf{r})|^{2}\left[1+e^{-\left(\gamma_{110}+\gamma_{001}\right) t}-\cos \left(2\left|\widetilde{\Omega}_{R}^{(1,1)}\right| t\right) e^{-\left(\gamma_{110}+\gamma_{001}\right) t}\right], \\
\left\langle\Psi\left|\hat{H}_{a}\right| \Psi\right\rangle=W \frac{1+\cos \left(2\left|\widetilde{\Omega}_{R}^{(1,1)}\right| t\right)}{2} e^{-\left(\gamma_{110}+\gamma_{001}\right) t}
\end{gathered}
$$

Fig. 4 illustrates the dynamics of observables in Eqs. (39) and (40).

Note that the Weisskopf-Wigner theory would give the same expression (40) for the atomic energy, but a wrong expression for the EM field intensity:

$$
\left\langle\Psi\left|\hat{\mathbf{E}}^{2}\right| \Psi\right\rangle=|\mathbf{E}(\mathbf{r})|^{2}\left[2-\cos \left(2\left|\widetilde{\Omega}_{R}^{(1,1)}\right| t\right)\right] e^{-\left(\gamma_{110}+\gamma_{001}\right) t}
$$

which does not approach the correct vacuum state. 


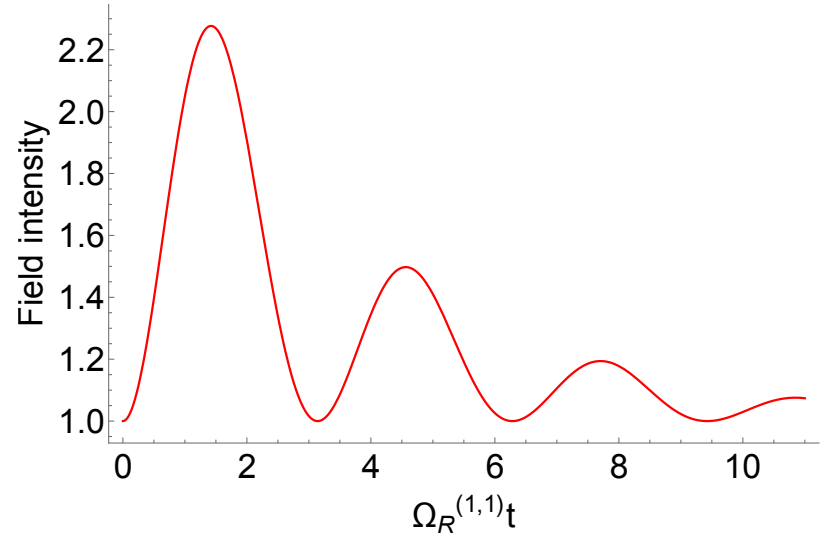

(a)

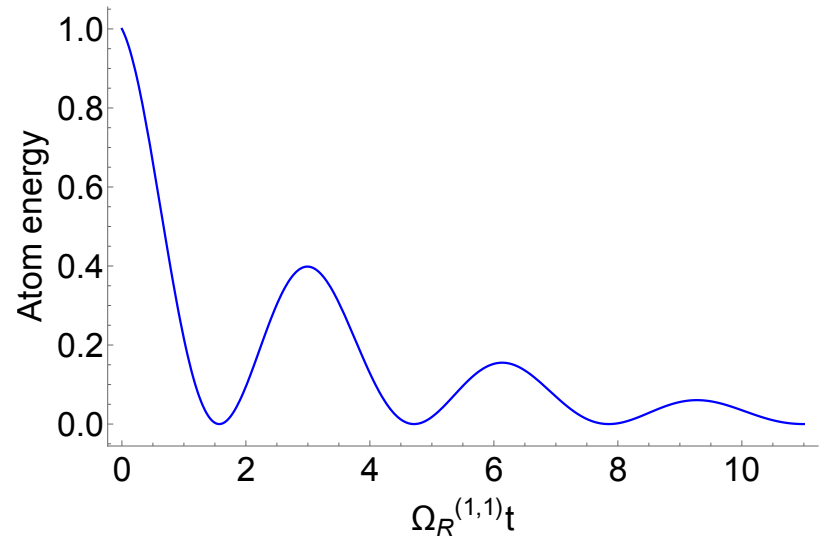

(b)

FIG. 4: (a) Normalized field intensity, $\left\langle\Psi\left|\hat{\mathbf{E}}^{2}\right| \Psi\right\rangle /|\mathbf{E}(\mathbf{r})|^{2}$, and (b) normalized atom energy $\left\langle\Psi\left|\hat{H}_{a}\right| \Psi\right\rangle / W$ as a function of time in units of the generalized Rabi frequency $\Omega_{R}^{(1,1)}$. Here $\gamma_{110}+\gamma_{001}=0.3 \Omega_{R}^{(1,1)}$.

\section{Emission spectra}

According to [23], the power spectrum of the emission is

$$
S(\boldsymbol{r}, \nu)=\frac{1}{\pi} \operatorname{Re} \int_{0}^{\infty} d \tau G^{(1)}(\boldsymbol{r}, \boldsymbol{r} ; \tau) e^{i \nu \tau},
$$

where $G^{(1)}(\boldsymbol{r}, \boldsymbol{r} ; \tau)$ is the field autocorrelation function at the position $\boldsymbol{r}$ of the detector:

$$
G^{(1)}(\boldsymbol{r}, \boldsymbol{r} ; \tau)=|\boldsymbol{E}(\boldsymbol{r})|^{2} \int_{0}^{\infty} d t \overline{\left\langle\hat{c}_{d}^{\dagger}(t) \hat{c}_{d}(t+\tau)\right\rangle} .
$$

where $\hat{c}_{d}(t), \hat{c}_{d}^{\dagger}(t)$ are annihilation and creation operators for the photons which interact with the detector, and the Heisenberg picture is used. We will assume that the coupling between 
the photons and the detector is weak, so the photon detection does not affect the dynamics of the intracavity photons. According to [32], $\hat{c}_{d}(t) \propto \hat{c}(t)$ for a nanocavity, so we can calculate the $G^{(1)}(\boldsymbol{r}, \boldsymbol{r} ; \tau)$ using operators for the cavity field $\hat{c}(t), \hat{c}^{\dagger}(t)$, up to a constant factor in the result. Note that the lower limit of the integral over $t$ is set to be $t=0$, which requires that no photons exist before $t=0$.

In the Heisenberg-Langevin approach, an operator in the Heisenberg picture can be expressed through Schrödinger's operators using the effective Hamiltonian $\hat{H}_{e f f}$, which contains the anti-Hermitian part; see the Appendix. At the same time, the inhomogeneous term proportional to the noise sources should be added. Including these noise terms in the solution for the field operators when calculating the emission spectra is equivalent to taking into account the detection of thermal radiation which seeps into the cavity from outside and spontaneous emission resulting from thermal excitation of an atom. We assume that the reservoir temperature in energy units is much lower than $W$ and $\hbar \omega$, so that the contribution of these noise terms to the emission spectra can be neglected (although noise is still needed to preserve the norm).

Then, the average correlator $\overline{\left\langle\hat{c}^{\dagger}(t) \hat{c}(t+\tau)\right\rangle}$ is expressed as

$$
\begin{aligned}
& \overline{\left\langle\hat{c}^{\dagger}(t) \hat{c}(t+\tau)\right\rangle} \\
& =\overline{\left\langle\Psi(t=0)\left|e^{i \hat{H}_{e f f}^{\dagger} t / \hbar} \hat{c}^{\dagger} e^{-i \hat{H}_{e f f} t / \hbar} e^{i \hat{H}_{e f f}^{\dagger}(t+\tau) / \hbar} \hat{c} e^{-i \hat{H}_{e f f}(t+\tau) / \hbar}\right| \Psi(t=0)\right\rangle} \\
& =\overline{\left\langle\Psi(t)\left|\hat{c}^{\dagger} e^{-i \hat{H}_{e f f} t / \hbar} e^{i \hat{H}_{e f f}^{\dagger}(t+\tau) / \hbar} \hat{c}\right| \Psi(t+\tau)\right\rangle},
\end{aligned}
$$

where $|\Psi(t)\rangle$ is the state vector of the system which we found in the previous subsection. It can be written as $|\Psi(t)\rangle=\sum_{n=0}^{\infty} C_{n}(t)|n\rangle\left|\Psi_{n}^{\alpha, e}(t)\right\rangle$, where $\left|\Psi_{n}^{\alpha, e}(t)\right\rangle$ is the part describing phonons and electrons. Therefore.

$$
\begin{aligned}
& \overline{\left\langle\hat{c}^{\dagger}(t) \hat{c}(t+\tau)\right\rangle} \\
& =\overline{\left(\sum _ { n = 0 } ^ { \infty } C _ { n } ^ { * } ( t ) \left\langlen\left|\left\langle\Psi_{n}^{\alpha, e}(t)\right|\right) \hat{c}^{\dagger} e^{-i \hat{H}_{e f f} t / \hbar} e^{i \hat{H}_{e f f}^{\dagger}(t+\tau) / \hbar} \hat{c}\left(\sum_{n=0}^{\infty} C_{n}(t+\tau)|n\rangle\left|\Psi_{n}^{\alpha, e}(t+\tau)\right\rangle\right)\right.\right.} \\
& =\overline{\left(\sum _ { n = 0 } ^ { \infty } \sqrt { n } C _ { n } ^ { * } ( t ) \left\langlen-1\left|\left\langle\Psi_{n}^{\alpha, e}(t)\right|\right) e^{-i \hat{H}_{e f f} t / \hbar} e^{i \hat{H}_{e f f}^{\dagger}(t+\tau) / \hbar}\left(\sum_{n=0}^{\infty} \sqrt{n} C_{n}(t+\tau)|n-1\rangle\left|\Psi_{n}^{\alpha, e}(t+\tau)\right\rangle\right)\right.\right.} .
\end{aligned}
$$

Consider a simple example when the initial state is $|0\rangle|0\rangle|1\rangle$. Within the RWA the system can only reach states $|0\rangle|0\rangle|1\rangle,|1\rangle|1\rangle|0\rangle$ and $|0\rangle|0\rangle|0\rangle$. After acting with $\hat{c}$ on a state of the 
system, a new state $|1\rangle|0\rangle|0\rangle$ can also appear, but it cannot evolve into other states. So, in this case we have

$$
\begin{aligned}
& \overline{\left\langle\hat{c}^{\dagger}(t) \hat{c}(t+\tau)\right\rangle} \\
& =\overline{\left(C _ { 1 } ^ { * } ( t ) \left\langle0\left|\left\langle\Psi_{1}^{\alpha, e}(t)\right|\right) e^{-i \hat{H}_{e f f} t / \hbar} e^{i \hat{H}_{e f f}^{\dagger}(t+\tau) / \hbar}\left(C_{1}(t+\tau)|0\rangle\left|\Psi_{1}^{\alpha, e}(t+\tau)\right\rangle\right)\right.\right.} \\
& =\overline{\left(C _ { 1 1 0 } ^ { * } ( t ) \left\langle1 \left|\left\langle0|\langle 0|) e^{-i \hat{H}_{e f f} t / \hbar} e^{i \hat{H}_{e f f}^{\dagger}(t+\tau) / \hbar}\left(C_{110}(t+\tau)|1\rangle|0\rangle|0\rangle\right)\right.\right.\right.\right.} \\
& =C_{110}^{*}(t) C_{110}(t+\tau) \exp \left[i \omega_{1,0} \tau-\gamma_{100}(2 t+\tau)\right],
\end{aligned}
$$

where we used Eqs. A32 and A33 and assumed that the noise for state $|1\rangle|0\rangle|0\rangle$ has zero correlator. Since

$$
C_{110}(t)=i \sin \left(\left|\tilde{\Omega}_{R}^{(1,1)}\right| t\right) \exp \left[-i \omega_{1,1} t-\frac{\gamma_{110}+\gamma_{001}}{2} t\right]
$$

we obtain

$$
\overline{\left\langle\hat{c}^{\dagger}(t) \hat{c}(t+\tau)\right\rangle}=\sin \left(\left|\tilde{\Omega}_{R}^{(1,1)}\right| t\right) \sin \left(\left|\tilde{\Omega}_{R}^{(1,1)}\right|(t+\tau)\right) \exp [-i \omega \tau] \exp \left[-\gamma_{\mathrm{ac}}(2 t+\tau)\right],
$$

where we introduced the notation $\gamma_{\mathrm{ac}} \equiv \gamma_{100}+\frac{\gamma_{110}+\gamma_{001}}{2}$. Then the power spectrum is found to be

$$
S(\boldsymbol{r}, \nu) \propto \frac{1}{\pi}|\boldsymbol{E}(\boldsymbol{r})|^{2} \frac{\left|\tilde{\Omega}_{R}^{(1,1)}\right|^{2}}{4 \gamma_{\mathrm{ac}}\left(\left|\tilde{\Omega}_{R}^{(1,1)}\right|^{2}+\gamma_{\mathrm{ac}}^{2}\right)} \operatorname{Re}\left[\frac{2 \gamma_{\mathrm{ac}}-i(\nu-\omega)}{\left[\gamma_{\mathrm{ac}}-i(\nu-\omega)\right]^{2}+\left|\tilde{\Omega}_{R}^{(1,1)}\right|^{2}}\right] .
$$

The normalized power spectra are shown in Fig. 5 for various values of $\left|\tilde{\Omega}_{R}^{(1,1)}\right| / \gamma_{\mathrm{ac}}$. For $\left|\tilde{\Omega}_{R}^{(1,1)}\right|<\gamma_{\text {ac }}$ the spectrum has a single maximum at zero detuning $\nu=\omega$. For $\left|\tilde{\Omega}_{R}^{(1,1)}\right|>\gamma_{\mathrm{ac}}$ the spectra are split and their maxima (same value for all spectra) are reached at detunings given by $(\nu-\omega)^{2}=\left|\tilde{\Omega}_{R}^{(1,1)}\right|^{2}-\gamma_{\text {ac }}^{2}$. Therefore, to reach the strong coupling regime the Rabi frequency $\left|\tilde{\Omega}_{R}^{(1,1)}\right|$ has to exceed the combination of the decoherence rates denoted by $\gamma_{\text {ac }}$.

\section{Relaxation rates}

Finally, we give explicit expressions for the relaxation constants $\gamma_{\alpha n 0}$ and $\gamma_{\alpha n 1}$. They were derived in Appendix using the Lindblad master equation approach and assuming statistical independence of "partial" dissipative reservoirs for the atomic, EM, and phonon subsystems. The result is

$$
\gamma_{\alpha n 0}=\frac{\gamma}{2} N_{1}^{T_{a}}+\frac{\mu_{\omega}}{2}\left[\bar{n}_{\omega}^{T_{e m}}(n+1)+\left(\bar{n}_{\omega}^{T_{e m}}+1\right) n\right]+\frac{\mu_{\Omega}}{2}\left[\bar{n}_{\Omega}^{T_{p}}(\alpha+1)+\left(\bar{n}_{\Omega}^{T_{p}}+1\right) \alpha\right]
$$




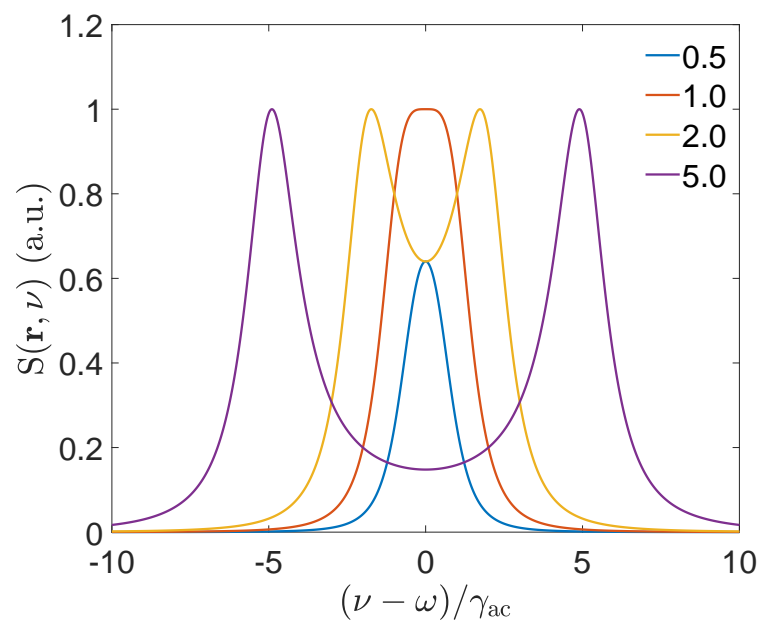

FIG. 5: The emission spectra for $\left|\tilde{\Omega}_{R}^{(1,1)}\right| / \gamma_{\text {ac }}$ equal to $0.5,1,2$ and 5. All spectra are normalized by the same constant.

$$
\gamma_{\alpha n 1}=\frac{\gamma}{2} N_{0}^{T_{a}}+\frac{\mu_{\omega}}{2}\left[\bar{n}_{\omega}^{T_{e m}}(n+1)+\left(\bar{n}_{\omega}^{T_{e m}}+1\right) n\right]+\frac{\mu_{\Omega}}{2}\left[\bar{n}_{\Omega}^{T_{p}}(\alpha+1)+\left(\bar{n}_{\Omega}^{T_{p}}+1\right) \alpha\right]
$$

where $\gamma, \mu_{\omega}$ and $\mu_{\Omega}$ are partial relaxation rates of the atomic, photon, and phonon subsystems respectively; $N_{0}^{T_{a}}=\frac{1}{1+e^{-\frac{W}{T_{a}}}}, N_{1}^{T_{a}}=\frac{e^{-\frac{W}{T_{a}}}}{1+e^{-\frac{W}{T_{a}}}}, \bar{n}_{\omega}^{T_{e m}}=\frac{1}{e^{\frac{\hbar \omega}{T_{e m}}}-1}, \bar{n}_{\Omega}^{T_{p}}=\frac{1}{e^{\frac{\hbar \Omega}{T_{p}}}-1}$ are their occupation numbers at thermal equilibrium; $T_{a, e m, p}$ are temperatures of partial atom, photon, and phonon reservoirs in energy units. As a reminder, the atom energy is equal to 0 in state $|0\rangle$ and $W$ in state $|1\rangle$.

If all reservoirs are at zero temperature, we obtain

$$
\gamma_{\alpha n 0}=\frac{\mu_{\omega}}{2} n+\frac{\mu_{\Omega}}{2} \alpha, \gamma_{\alpha n 1}=\frac{\gamma}{2}+\frac{\mu_{\omega}}{2} n+\frac{\mu_{\Omega}}{2} \alpha
$$

Eq. (51) shows that $\gamma_{000}=0$, validating our choice earlier in this section. We also obtain physically intuitive expressions for $\gamma_{110}$ and $\gamma_{001}: \gamma_{110}=\frac{\mu_{\omega}}{2}+\frac{\mu_{\Omega}}{2}, \gamma_{001}=\frac{\gamma}{2}$.

\section{CLASSICAL ACOUSTIC PUMPING}

In this case the RWA Hamiltonian is given by Eq. (16). It depends only on quantum operators $\hat{\sigma}, \hat{\sigma}^{\dagger}$ and $\hat{c}, \hat{c}^{\dagger}$; therefore the state vector has to be expanded over the basis states $|n\rangle|0\rangle$ and $|n\rangle|1\rangle$ :

$$
\Psi=\sum_{n=0}^{\infty}\left(C_{n 0}|n\rangle|0\rangle+C_{n 1}|n\rangle|1\rangle\right) .
$$


Substituting Eq. (52) in the Schrödinger equation with the Hamiltonian (16), we again get separation into a block of two equations,

$$
\begin{gathered}
\dot{C}_{n 0}=-i \omega_{n} C_{n 0}+i \frac{\mathfrak{R}^{*}}{\hbar} e^{i \Omega t} C_{(n-1) 1} \sqrt{n} \\
\dot{C}_{(n-1) 1}=-i\left(\omega_{n-1}+\frac{W}{\hbar}\right) C_{(n-1) 1}+i \frac{\mathfrak{R}}{\hbar} e^{-i \Omega t} C_{n 0} \sqrt{n}
\end{gathered}
$$

and a separate equation for the amplitude of the ground state $|0\rangle|0\rangle$ of the system:

$$
\dot{C}_{00}=-i \omega_{0} C_{00}
$$

where $\omega_{n}=\omega\left(n+\frac{1}{2}\right)$. After making the substitution $C_{(n-1) 1}=G_{(n-1) 1} e^{-i \Omega t}$, Eqs. (53), 54 give the equations similar in form to Eqs. (19):

$$
\frac{d}{d t}\left(\begin{array}{c}
C_{n 0} \\
G_{(n-1) 1}
\end{array}\right)+\left(\begin{array}{cc}
i \omega_{n} & -i \Omega_{R}^{(n) *} \\
-i \Omega_{R}^{(n)} & i \omega_{n}-i \Delta
\end{array}\right)\left(\begin{array}{c}
C_{n 0} \\
G_{(n-1) 1}
\end{array}\right)=0
$$

where

$$
\Omega_{R}^{(n)}=\frac{\Re}{\hbar} \sqrt{n}, \Delta=\Omega+\omega-\frac{W}{\hbar}, \omega_{n}-\Delta=\omega_{n-1}+\frac{W}{\hbar} .
$$

Eqs. (55), (56) are different from Eqs. (19), 20) only in one aspect: they don't contain the index of the quantum state of the phonon field, whereas the Rabi frequency depends on the amplitude of classical acoustic oscillations $\mathbf{Q}\left(\mathbf{r}_{a}\right)$, see Sec. IIE. Obviously, the solution to Eqs. (55), (56) will have the same form and the expressions (26), 27) for the observables will remain the same, after dropping the index of the quantum phonon state and redefining the Rabi frequency.

Dissipation due to coupling to a reservoir can be included using the stochastic equation of evolution of the state vector, see the Appendix. The corresponding equations are again similar to those for a fully quantum problem given by Eqs. (28), 29):

$$
\begin{gathered}
\dot{C}_{00}+i\left(\omega_{0}+\gamma_{00}\right) C_{00}=-\frac{i}{\hbar} R_{00} \\
\frac{d}{d t}\left(\begin{array}{c}
C_{n 0} \\
C_{(n-1) 1}
\end{array}\right)+\left(\begin{array}{cc}
i \omega_{n}+\gamma_{n 0} & -i \Omega_{R}^{(n) *} \\
-i \Omega_{R}^{(n)} & i \omega_{n}-i \Delta+\gamma_{(n-1) 1}
\end{array}\right)\left(\begin{array}{c}
C_{n 0} \\
C_{(n-1) 1}
\end{array}\right)=-\frac{i}{\hbar}\left(\begin{array}{c}
R_{n 0} \\
R_{(n-1) 1}
\end{array}\right) .
\end{gathered}
$$

Since the acoustic field is now a given external pumping, the relaxation constants should not depend on the parameters of a phonon reservoir. They can be obtained after obvious simplification of Eqs. 49, 50): 


$$
\begin{aligned}
& \gamma_{n 0}=\frac{\gamma}{2} N_{1}^{T_{a}}+\frac{\mu_{\omega}}{2}\left[\bar{n}_{\omega}^{T_{e m}}(n+1)+\left(\bar{n}_{\omega}^{T_{e m}}+1\right) n\right], \\
& \gamma_{n 1}=\frac{\gamma}{2} N_{0}^{T_{a}}+\frac{\mu_{\omega}}{2}\left[\bar{n}_{\omega}^{T_{e m}}(n+1)+\left(\bar{n}_{\omega}^{T_{e m}}+1\right) n\right],
\end{aligned}
$$

All expressions for the state vector and observables can be obtained from the corresponding expressions in Sec. IV after dropping the index $\alpha$ of the quantum state of the phonon field and redefining the frequency of Rabi oscillations.

\section{SEPARATION AND INTERPLAY OF THE PARAMETRIC AND ONE- PHOTON RESONANCE}

For an electron system coupled to a EM cavity mode and dressed by a phonon field, the phonon frequency $\Omega$ can be much lower than the optical frequency. In this case the overlap of the parametric (three-wave) resonance $\omega \pm \Omega \approx \frac{W}{\hbar}$ and the one-photon (two-wave) resonance $\omega \approx \frac{W}{\hbar}$ can be an issue.

First of all, it is clear that the resonances can be separated only if the value of $\Omega$ exceeds the sum of the spectral widths of the EM cavity mode and the electron transition.

Second, the separation criterion imposes certain restrictions on the Rabi frequencies of the two resonances. To derive these restrictions, we neglect dissipation and retain in the Hamiltonian (11) both the RWA terms near the parametric resonance $\omega \pm \Omega \approx \frac{W}{\hbar}$, and the terms near a one-photon resonance $\omega \approx \frac{W}{\hbar}$. Since the result will be almost the same whether the phonon field is quantized or classical, we will consider the classical phonon field to keep the expressions a bit shorter. The resulting Hamiltonian is

$$
\hat{H}=\hbar \omega\left(\hat{c}^{\dagger} \hat{c}+\frac{1}{2}\right)+W \hat{\sigma}^{\dagger} \hat{\sigma}-\left(\chi+\mathfrak{R} e^{-i \Omega t}\right) \hat{\sigma}^{\dagger} \hat{c}-\left(\chi^{*}+\mathfrak{R}^{*} e^{i \Omega t}\right) \hat{\sigma} \hat{c}^{\dagger},
$$

where $\chi=(\mathbf{d} \cdot \mathbf{E})_{\mathbf{r}=\mathbf{r}_{a}}, \mathfrak{R}=[\mathbf{d}(\mathbf{Q} \cdot \nabla) \mathbf{E}]_{\mathbf{r}=\mathbf{r}_{a}} ; \mathbf{Q}$ is now a complex-valued amplitude of classical phonon oscillations. The value of $\Omega$ in Eq. (61) can be both positive and negative, corresponding to the choice of an upper or lower sign in the parametric resonance condition $\omega \pm \Omega \approx \frac{W}{\hbar}$. The change of sign in $\Omega$ corresponds to replacing $\mathbf{Q}$ with $\mathbf{Q}^{*}$ in the expression for $\mathfrak{R}$.

The state vector should be sought in the form of Eq. (52). After substituting it into the Schrödinger equation we obtain coupled equations for the amplitudes of basis states $|n\rangle|0\rangle$, 
$|n-1\rangle|1\rangle:$

$$
\begin{aligned}
& \dot{C}_{n 0}+i \omega_{n} C_{n 0}-\frac{i}{\hbar}\left(\chi^{*}+\mathfrak{R}^{*} e^{i \Omega t}\right) \sqrt{n} C_{(n-1) 1}=0, \\
& \dot{C}_{(n-1) 1}+i\left(\omega_{n-1}+\frac{W}{\hbar}\right) C_{(n-1) 1}-\frac{i}{\hbar}\left(\chi+\mathfrak{R} e^{-i \Omega t}\right) \sqrt{n} C_{n 0}=0
\end{aligned}
$$

and

$$
\dot{C}_{00}+i \omega_{0} C_{00}=0
$$

where $\omega_{n}=\omega\left(n+\frac{1}{2}\right)$. To compare these equations with Eqs. (53) and (54), it is convenient to assume that the system is exactly at one of the resonances and study the behavior of the solution with increasing the detuning from another resonance. For example, we assume an exact parametric resonance $\omega+\Omega=\frac{W}{\hbar}$. In this case the detuning from the two-wave resonance is $\frac{W}{\hbar}-\omega=\Omega$. After the substitution $C_{n 0}=G_{n 0} e^{-i \omega_{n} t}$ and $C_{(n-1) 1}=$ $G_{(n-1) 1} e^{-i\left(\omega_{n-1}+\frac{W}{\hbar}\right) t}$, we obtain from Eqs. 62 and 63 that

$$
\begin{aligned}
& \dot{G}_{n 0}-\frac{i}{\hbar}\left(\chi^{*}+\mathfrak{R}^{*} e^{i \Omega t}\right) \sqrt{n} G_{(n-1) 1} e^{-i\left(\frac{W}{\hbar}-\omega\right) t}=0, \\
& \dot{G}_{(n-1) 1}-\frac{i}{\hbar}\left(\chi+\mathfrak{R} e^{-i \Omega t}\right) \sqrt{n} G_{n 0} e^{i\left(\frac{W}{\hbar}-\omega\right) t}=0 .
\end{aligned}
$$

If we neglect at first the perturbation of the system in the vicinity of the two-wave resonance, the solution to Eqs. (65) and (66) at $\chi=0$ is

$$
\left(\begin{array}{c}
G_{n 0} \\
G_{(n-1) 1}
\end{array}\right)=A e^{i \Omega_{R}^{(3)} t}\left(\begin{array}{l}
1 \\
1
\end{array}\right)+B e^{-i \Omega_{R}^{(3)} t}\left(\begin{array}{c}
1 \\
-1
\end{array}\right)
$$

where $\Omega_{R}^{(3)}=\frac{1}{\hbar} \Re \sqrt{n}$ is the Rabi frequency of the parametric resonance, $A$, and $B$ are arbitrary constants. The state described by Eq. (67) is obviously entangled.

To write the formal solution to Eqs. (65) and (66), we make another substitution of variables: $G_{n 0} \pm G_{(n-1) 1}=G_{ \pm}$. The result is

$$
\dot{G}_{ \pm} \mp i \Omega_{R}^{(3)} G_{ \pm}=i \Omega_{R}^{(2) *} e^{-i \Omega t} G_{n 0} \pm i \Omega_{R}^{(2)} e^{i \Omega t} G_{(n-1) 1}
$$

where $\Omega_{R}^{(2)}=\frac{1}{\hbar} \chi \sqrt{n}$ is the Rabi frequency corresponding to the one-photon (two-wave) resonance. The solution to the last equation is

$$
G_{ \pm}=2(A, B) e^{ \pm i \Omega_{R}^{(3)} t}+i e^{ \pm i \Omega_{R}^{(3)} t} \int_{0}^{t} e^{\mp i \Omega_{R}^{(3)} \tau}\left[\Omega_{R}^{(2) *} e^{-i \Omega \tau} G_{n 0}(\tau) \pm \Omega_{R}^{(2)} e^{i \Omega \tau} G_{(n-1) 1}(\tau)\right] d \tau
$$


Considering the terms proportional to $\Omega_{R}^{(2)}$ as perturbation, we seek the solution as

$$
\left(\begin{array}{c}
G_{n 0} \\
G_{(n-1) 1}
\end{array}\right)=A e^{i \Omega_{R}^{(3)} t}\left(\begin{array}{l}
1 \\
1
\end{array}\right)+B e^{-i \Omega_{R}^{(3)} t}\left(\begin{array}{c}
1 \\
-1
\end{array}\right)+\left(\begin{array}{c}
\delta G_{n 0} \\
\delta G_{(n-1) 1}
\end{array}\right) .
$$

To estimate the magnitude of the perturbation, we substitute Eq. 67) into Eq. 68). After some algebra we obtain that under the condition $\Omega_{R}^{(3)} \ll \Omega$ the magnitude of the perturbation is

$$
\delta G_{n 0,(n-1) 1} \sim\left|\frac{\Omega_{R}^{(2)}}{\Omega}\right| G_{n 0,(n-1) 1},
$$

whereas if $\Omega_{R}^{(3)} \sim \Omega$ the magnitude of the perturbation is

$$
\delta G_{n 0,(n-1) 1} \sim\left|\frac{\Omega_{R}^{(2)}}{\Omega_{R}^{(3)}}\right| G_{n 0,(n-1) 1} .
$$

To summarize this part, if both Rabi frequencies $\Omega_{R}^{(3)}, \Omega_{R}^{(2)} \ll \Omega$, the two resonances can be treated independently for any relationship between the magnitudes of $\Omega_{R}^{(3)}$ and $\Omega_{R}^{(2)}$. If the above inequality is violated, one can neglect one of the resonances only if its associated Rabi frequency is much lower than the Rabi frequency of another resonance. These restrictions are obvious from qualitative physical reasoning: either the magnitudes of the Rabi splittings are much smaller than the distance between resonances, or one of the splittings is much weaker than another one.

When the effect of the neighboring resonance is non-negligible, it can still be taken into account in the solution. Indeed, consider the solution to Eqs. (62) and (63), taking into account only the two-wave resonance, i.e. taking $\mathfrak{R}=0$. After obvious substitutions, we arrive at

$$
\begin{aligned}
\left(\begin{array}{c}
C_{n 0} \\
C_{(n-1) 1}
\end{array}\right)= & A e^{-i\left(\omega_{n}-\frac{\Omega}{2}+\sqrt{\frac{\Omega^{2}}{4}+\left|\Omega_{R}^{(2)}\right|^{2}}\right) t} \times\left(\begin{array}{c}
1 \\
\frac{-\frac{\Omega}{2}+\sqrt{\frac{\Omega^{2}}{4}+\left|\Omega_{R}^{(2)}\right|^{2}}}{\Omega_{R}^{(2)}}
\end{array}\right) \\
& +B e^{-i\left(\omega_{n-1}+\frac{W}{\hbar}+\frac{\Omega}{2}-\sqrt{\frac{\Omega^{2}}{4}+\left|\Omega_{R}^{(2)}\right|^{2}}\right) t} \times\left(\begin{array}{c}
\frac{\Omega_{R}^{(2)}}{-\frac{\Omega}{2}-\sqrt{\frac{\Omega^{2}}{4}+\left|\Omega_{R}^{(2)}\right|^{2}}} \\
1
\end{array}\right) ;
\end{aligned}
$$

In the limit $\Omega \gg \Omega_{R}^{(2)}$ we obtain

$$
\left(\begin{array}{c}
C_{n 0} \\
C_{(n-1) 1}
\end{array}\right) \approx A e^{-i\left(\omega_{n}+\frac{\left|\Omega_{R}^{(2)}\right|^{2}}{\Omega}\right) t}\left(\begin{array}{c}
1 \\
\frac{\left|\Omega_{R}^{(2)}\right|}{\Omega}
\end{array}\right)+B e^{-i\left(\omega_{n-1}+\frac{W}{\hbar}-\frac{\left|\Omega_{R}^{(2)}\right|^{2}}{\Omega}\right) t}\left(\begin{array}{c}
-\frac{\left|\Omega_{R}^{(2)}\right|}{\Omega} \\
1
\end{array}\right)
$$


It is clear from Eq. (70) that the entanglement of states described by $C_{n 0}$ and $C_{(n-1) 1}$ is determined by a small parameter $\frac{\left|\Omega_{R}^{(2)}\right|}{\Omega}$, whereas at exact resonance the entanglement is always stronger; see Eq. (67). Therefore, when $\Omega_{R}^{(2)} \ll \Omega$, we can neglect the contribution of the two-photon resonance to the entanglement of states $|n\rangle|0\rangle$ and $|n-1\rangle|1\rangle$. However, it follows from Eq. (69) that the two-wave resonance shifts the eigenfrequencies of the system. Qualitatively, these shifts can be included by putting $\chi=0$ in Eqs. (62) and (63) but replacing the eigenfrequencies $\omega_{n}$ and $\omega_{n-1}$ according to Eq. (70):

$$
\omega_{n} \Longrightarrow \omega_{n}+\frac{\left|\Omega_{R}^{(2)}\right|^{2}}{\Omega}, \omega_{n-1}+\frac{W}{\hbar} \Longrightarrow \omega_{n-1}+\frac{W}{\hbar}-\frac{\left|\Omega_{R}^{(2)}\right|^{2}}{\Omega} .
$$

If $\Omega_{R}^{(3)} \ll \frac{\left|\Omega_{R}^{(2)}\right|^{2}}{\Omega}$ these shifts can be significant in order to interpret the spectra near the three-wave parametric resonance.

The same reasoning can be carried out to analyze the effect of a detuned three-wave resonance on the solution near the two-wave resonance.

These results can be verified by an exact numerical solution of Eqs. (62) and (63) for given initial conditions. After that, we can obtain the spectra of $C_{n 0}$ and $C_{(n-1) 1}$. Since they are oscillating functions, their spectra form discrete lines at frequencies which we denote as $\omega_{\text {osc }}$.

As an example, we select the case of $n=1$, set $\left|\Omega_{R}^{(2)}\right|=\left|\Omega_{R}^{(3)}\right|=0.1 \Omega$, and choose the initial condition as $C_{n 0}(0)=0$ and $C_{(n-1) 1}(0)=1$. The frequencies $\omega_{\text {osc }}$ of the spectral lines for $C_{n 0}$ and $C_{(n-1) 1}$ are shown in Fig. 6. Their values are shifted by $\omega_{o s c, 0}=\left.\omega_{1}\right|_{\omega=W / \hbar}$. The area of the dot for each spectral line is proportional to the square of its amplitude. If a marker is not visible, it means the corresponding line is very weak and can be neglected. The anticrossing can be seen at both the one-photon resonance and parametric resonance.

As an illustration of the violation of the condition for resonance separation, we show the oscillation frequencies for $\left|\Omega_{R}^{(2)}\right|=\left|\Omega_{R}^{(3)}\right|=0.5 \Omega$ in Fig. 7. Here the anticrossing picture of isolated resonances is smeared and cannot be observed.

\section{CONTROL OF ENTANGLED STATES}

In order to control the quantum state of the system, turn the entanglement on/off, read or write information into a qubit, or implement a logic gate, one has to vary the parameters of a system, for example the detuning from resonance, the field amplitude of the EM mode 

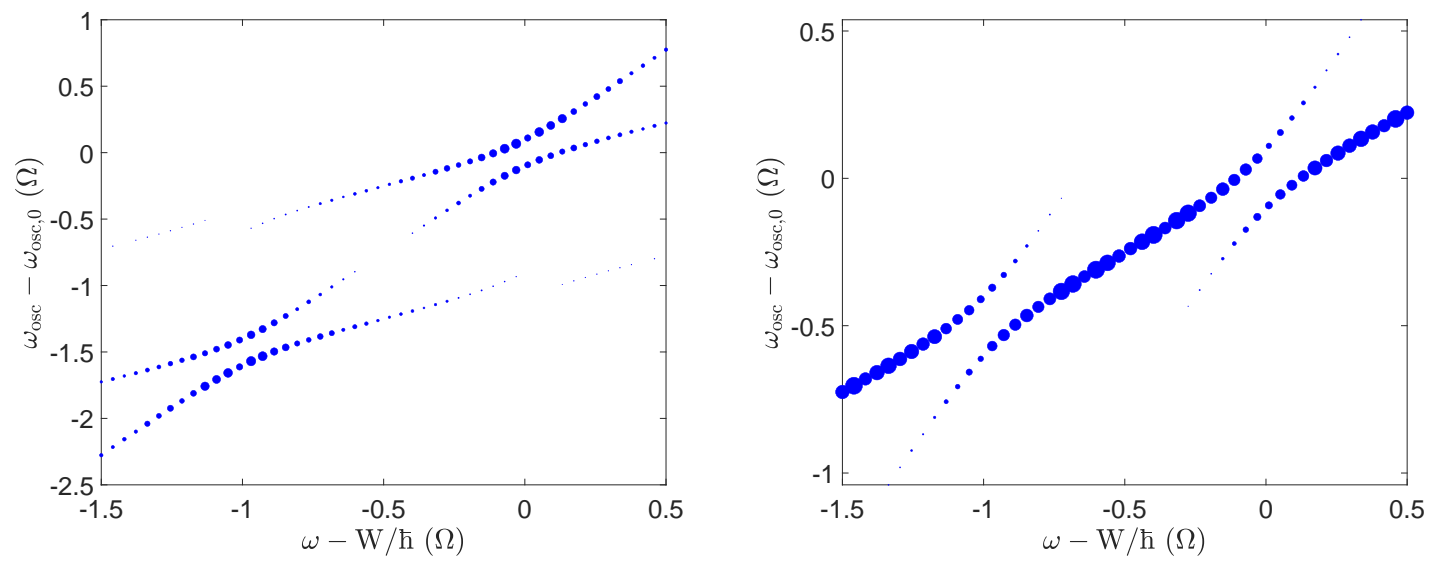

FIG. 6: The frequencies $\omega_{\text {osc }}$ of the spectral lines for $C_{n 0}$ (left panel), and $C_{(n-1) 1}$ (right panel), with $n=1$, as functions of the photon frequency $\omega$. The photon frequencies are shifted by $W / \hbar$, and the positions of spectral lines $\omega_{\text {osc }}$ are shifted by $\omega_{o s c, 0}=\left.\omega_{1}\right|_{\omega=W / \hbar}$. The area of a marker is proportional to the amplitude squared of the spectral line. Both axes are in units of $\Omega$. The parameters are $\left|\Omega_{R}^{(2)}\right|=\left|\Omega_{R}^{(3)}\right|=0.1 \Omega$, and the initial condition is $C_{n 0}(0)=0$ and $C_{(n-1) 1}(0)=1$.
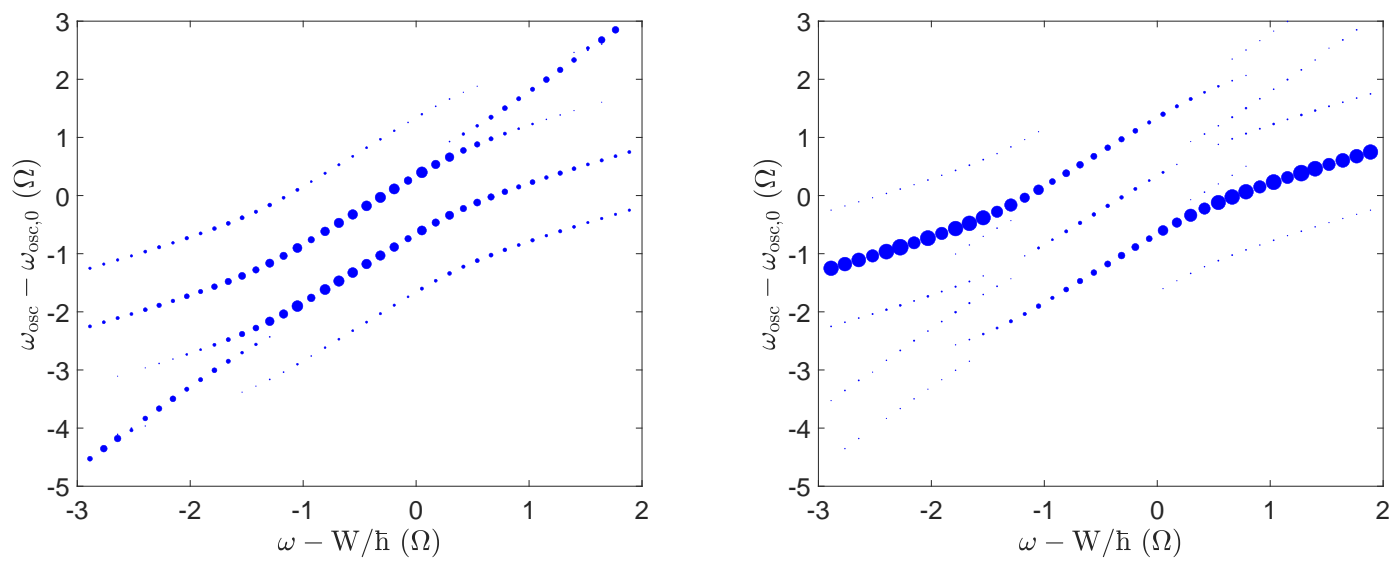

FIG. 7: The frequencies $\omega_{\text {osc }}$ of the spectral lines for $C_{n 0}$ (left panel), and $C_{(n-1) 1}$ (right panel), with $n=1$. The notations are the same as in Fig. 6. The parameters are $\left|\Omega_{R}^{(2)}\right|=\left|\Omega_{R}^{(3)}\right|=0.5 \Omega$, and the initial condition is $C_{n 0}(0)=0$ and $C_{(n-1) 1}(0)=1$.

at the atom position, or the intensity of a classical acoustic pumping. The analytic results obtained in previous sections can be readily generalized when the variation of a parameter is adiabatic, i.e. slower than the optical frequencies $\omega$ or $\frac{W}{\hbar}$. Since the space is limited, the time-dependent problem will be considered elsewhere. Here we consider just one example, 
namely turning on/off of a classical acoustic pumping $\mathbf{q}=\mathbf{Q}(\mathbf{r}) e^{-i \Omega t}+\mathbf{Q}^{*}(\mathbf{r}) e^{i \Omega t}$.

For maximal control it is beneficial to place an atom at the point where $\mathbf{E}\left(\mathbf{r}=\mathbf{r}_{a}\right) \rightarrow 0$, whereas $(\mathbf{Q} \cdot \nabla) \mathbf{E}_{\mathbf{r}=\mathbf{r}_{a}}$ is maximized. The equations of motion for quantum state amplitudes were derived in Sec. V, see Eqs. (53)-(55).

Consider an exact parametric resonance $\omega+\Omega=\frac{W}{\hbar}$ for simplicity, when

$$
\omega_{n}=\omega_{n-1}+\frac{W}{\hbar} \text {. }
$$

The solution to Eqs. (53)-(55) when the acoustic pumping is turned off is

$$
\Psi=C_{00}(0) e^{-i \omega_{0} t}|0\rangle|0\rangle+\sum_{n=1}^{\infty}\left(C_{n 0}(0) e^{-i \omega_{n} t}|n\rangle|0\rangle+C_{(n-1) 1}(0) e^{-i\left(\omega_{n-1}+\frac{W}{\hbar}\right) t}|n-1\rangle|1\rangle\right)
$$

The solution when the acoustic pumping is turned on is

$$
\begin{aligned}
\Psi= & C_{00}(0) e^{-i \omega_{0} t}|0\rangle|0\rangle+\sum_{n=1}^{\infty}\left[\left(A_{n} e^{-i\left|\Omega_{R}^{(n)}\right| t}+B_{n} e^{i\left|\Omega_{R}^{(n)}\right| t}\right) e^{-i \omega_{n} t}|n\rangle|0\rangle\right. \\
& \left.+\left(-A_{n} e^{-i\left|\Omega_{R}^{(n)}\right| t}+B_{n} e^{i\left|\Omega_{R}^{(n)}\right| t}\right) e^{i \theta-i\left(\omega_{n-1}+\frac{W}{\hbar}\right) t}|n-1\rangle|1\rangle\right]
\end{aligned}
$$

Assume that the initial quantum state before the pumping was turned on was not entangled, for example, an atom was in an excited state and there were no photons:

$$
\Psi=e^{-i\left(\omega_{0}+\frac{W}{\hbar}\right) t}|0\rangle|1\rangle
$$

If the acoustic pumping is turned on at $t=0$, the quantum state becomes entangled:

$$
\Psi=i e^{-i \omega_{1} t-i \theta} \sin \left(\left|\Omega_{R}^{(1)}\right| t\right)|1\rangle|0\rangle+e^{-i\left(\omega_{0}+\frac{W}{\hbar}\right) t} \cos \left(\left|\Omega_{R}^{(1)}\right| t\right)|0\rangle|1\rangle,
$$

Then the acoustic pumping can be turned off. Depending on the turnoff moment of time, one can obtain various entangled photon-atom states, e.g. Bell states etc. The above reasoning is valid when the turn-on/off rate is slower than the optical frequencies and the detuning from the two-wave resonance $\omega=\frac{W}{\hbar}$.

\section{CONCLUSIONS}

In conclusion, we showed how the entanglement in a system of a fermionic quantum emitter coupled to a quantized electromagnetic field in a nanocavity and quantized phonon or mechanical vibrational modes emerges in the vicinity of a parametric resonance in the 
system. We developed analytic theory describing the formation and evolution of entangled quantum states, which can be applied to a broad range of cavity quantum optomechanics problems and emerging nanocavity strong-coupling experiments. The model includes decoherence effects due to coupling of the fermion, photon, and phonon subsystems to their dissipative reservoirs within the stochastic evolution approach, which is derived from the Heisenberg-Langevin formalism. We showed that our approach provided the results for physical observables equivalent to those obtained from the density matrix equations with the relaxation operator in Lindblad form. We derived analytic expressions for the time evolution of the quantum state and observables, and the emission spectra. The limit of a classical acoustic pumping, the control of entangled states, and the interplay between parametric and standard two-wave resonances were discussed.

\section{ACKNOWLEDGMENTS}

This work has been supported in part by the Air Force Office for Scientific Research through Grant No. FA9550-17-1-0341 and by NSF Award No. 1936276. M.T. acknowledges the support from RFBR Grant No. 20-02-00100, M.E. acknowledges the support from Federal Research Center Institute of Applied Physics of the Russian Academy of Sciences (Project No. 0035-2019-004).

\section{Appendix A: The stochastic equation of evolution for the state vector}

The description of open quantum systems within the stochastic equation of evolution for the state vector is usually formulated for a Monte-Carlo type numerical scheme, e.g. the method of quantum jumps [23, 25]. We developed an approach suitable for analytic derivations. Our stochastic equation of evolution is basically the Schrödinger equation modified by adding a linear relaxation operator and the noise source term with appropriate correlation properties. The latter are related to the parameters of the relaxation operator in such a way that the expressions for the statistically averaged quantities satisfy certain physically meaningful conditions.

The protocol of introducing the relaxation operator with a corresponding noise source term to the quantum dynamics is well known in the Heisenberg picture, where it is called the 
Heisenberg-Langevin method [23, 27, 28]. We develop a conceptually similar approach for the Schrödinger equation. Here we derive the general form of the stochastic equation of evolution from the Heisenberg-Langevin equations and track how certain physically reasonable constraints on the observables determine the correlation properties of the noise sources.

\section{From Heisenberg-Langevin equations to the stochastic equation for the state} vector

The Heisenberg-Langevin equation for the operator $\widehat{g}$ of a certain observable quantity takes the form [23, 27, 28]

$$
\frac{d}{d t} \hat{g}=\frac{i}{\hbar}[\hat{H}, \hat{g}]+\hat{R}(\hat{g})+\hat{L}_{g}(t),
$$

where $\hat{R}(\hat{g})$ is the relaxation operator, $\hat{L}_{g}(t)$ is the Langevin noise source satisfying $\overline{\hat{L}_{g}(t)}=$ 0 , where the bar means statistical averaging. For given commutation relations of the two operators: $\left[\hat{g}_{1}, \hat{g}_{2}\right]=C$, where $C$ is a constant, correct Langevin sources should ensure the conservation of commutation relations at any moment of time, despite the presence of the relaxation operator in Eq. (A1); see [28 30].

The group of terms $\frac{i}{\hbar}[\hat{H}, \hat{g}]+\hat{R}(\hat{g})$ can often be written as

$$
\frac{i}{\hbar}[\hat{H}, \hat{g}]+\hat{R}(\hat{g})=\frac{i}{\hbar}\left(\hat{H}_{e f f}^{\dagger} \hat{g}-\hat{g} \hat{H}_{e f f}\right),
$$

where $\hat{H}_{e f f}$ is a non-Hermitian operator. For example, if the relaxation operator describes dissipation with relaxation constant $\gamma$, so that $\overline{\hat{g}} \propto e^{-\gamma t}$, then $\hat{H}_{e f f}=\hat{H}-i \hbar \frac{\gamma}{2} \hat{1}$, where $\hat{1}$ is a unit operator. Note that in the master equation for the density matrix the relaxation is often introduced in a conceptually similar way [25], $[\hat{H}, \hat{\rho}] \Longrightarrow \hat{H}_{\text {eff }} \hat{\rho}-\hat{\rho} \hat{H}_{\text {eff }}^{\dagger}$, which is however slightly different from the form used in Eq. $\left[\right.$ A2): $[\hat{H}, \hat{g}] \Longrightarrow \hat{H}_{\text {eff }}^{\dagger} \hat{g}-\hat{g} \hat{H}_{\text {eff }}$. The difference is because the commutator of an unknown operator with Hamiltonian enters with opposite sign in the master equation as compared to the Heisenberg equation.

Now consider the transition from the Heisenberg-Langevin equation to the stochastic equation for the state vector. The key point is to assume that there exists the operator of evolution $\hat{U}(t)$, which is determined not only by the system parameters but also by the properties of the reservoir. This operator determines the evolution of the state vector:

$$
|\Psi(t)\rangle=\hat{U}(t)\left|\Psi_{0}\right\rangle,\langle\Psi(t)|=\left\langle\Psi_{0}\right| \hat{U}^{\dagger}(t),
$$


where $\Psi_{0}=\Psi(0)$. Hereafter we will denote the operators in the Schrödinger picture with index "s" to distinguish them from the Heisenberg operators. An observable can be calculated as

$$
g(t)=\left\langle\Psi(t)\left|\hat{g}_{S}\right| \Psi(t)\right\rangle=\left\langle\Psi_{0}|\hat{g}(t)| \Psi_{0}\right\rangle
$$

Which leads to

$$
\hat{g}(t)=\hat{U}^{\dagger}(t) \hat{g}_{S} \hat{U}(t)
$$

Since the substitution of Eqs. A3 and (A4) into the standard Heisenberg equation leads to the standard Schrödinger equation, it makes sense to apply the same procedure to the Heisenberg-Langevin equation in order to obtain the "stochastic variant" of the Schrödinger equation. The solution of the latter should yield the expression for an observable,

$$
g(t)=\overline{\left\langle\Psi(t)\left|\hat{g}_{S}\right| \Psi(t)\right\rangle}
$$

Which is different from the standard expression by additional averaging over the noise statistics.

Note that an open system interacting with a reservoir is generally in a mixed state and should be described by the density matrix. We are describing the state of the system with a state vector which has a fluctuating component. For example, in a certain basis $|\alpha\rangle$ the state vector will be $C_{\alpha}(t)=\overline{C_{\alpha}}+\widetilde{C_{\alpha}}$, where the fluctuating component is denoted with a wavy bar. The elements of the density matrix of the corresponding mixed state are $\rho_{\alpha \beta}=\overline{C_{\alpha} C_{\beta}^{*}}=\overline{C_{\alpha}} \cdot \overline{C_{\beta}^{*}}+\overline{{\widetilde{C_{\alpha}}} \cdot{\widetilde{C_{\beta}}}^{*}}$.

The solution to the Heisenberg-Langevin equation can be expressed through the evolution operator $\hat{U}(t)$ using Eq. A4). The noise source terms should be chosen to ensure the conservation of commutation relations at any moment of time, despite the presence of the relaxation operator. Since commutation relations between any two operators are conserved if and only if the evolution operator $\hat{U}(t)$ is unitary, a correct noise source in the HeisenbergLangevin equation will automatically ensure the condition $\hat{U}^{\dagger} \hat{U}=\hat{1}$.

We implement the above protocol. Substituting Eq. A4 together with $\hat{H}_{e f f}=\hat{U}^{\dagger} \hat{H}_{e f f, S} \hat{U}$ and $\hat{H}_{e f f}^{\dagger}=\hat{U}^{\dagger} \hat{H}_{e f f, S}^{\dagger} \hat{U}$ into Eqs. A1, A2, and using $\hat{U}^{\dagger} \hat{U}=\hat{1}$, we arrive at

$$
\left(\frac{d}{d t} \hat{U}^{\dagger}-\frac{i}{\hbar} \hat{U}^{\dagger} \hat{H}_{e f f, S}^{\dagger}\right) \hat{g}_{S} \hat{U}+\hat{U}^{\dagger} \hat{g}_{S}\left(\frac{d}{d t} \hat{U}+\frac{i}{\hbar} \hat{H}_{e f f, S} \hat{U}\right)=\hat{L}_{g}
$$

Next, we introduce the operator $\hat{F}$, defined by

$$
\hat{L}_{g}=2 \hat{U}^{\dagger} \hat{g}_{S} \hat{F}
$$


For the operator $\hat{L}_{g}^{\dagger}$, Eq. A6 gives $\hat{L}_{g}^{\dagger}=2 \hat{F}^{\dagger}\left(\hat{U}^{\dagger} \hat{g}_{S}\right)^{\dagger}=2 \hat{F}^{\dagger} \hat{g}_{S}^{\dagger} \hat{U}$. Since $\hat{g}$ and $\hat{g}_{S}$ are Hermitian operators, $\hat{L}_{g}$ has to be Hermitian too. (One can develop the Heisenberg-Langevin formalism for non-Hermitian operators too, for example creation or annihilation operators, but the derivation becomes longer.) Then the operator $\hat{L}_{g}$ can be "split" between the two terms on the left-hand side of Eq. A5 using the relationship

$$
\hat{L}_{g}=\hat{U}^{\dagger} \hat{g}_{S} \hat{F}+\hat{F}^{\dagger} \hat{g}_{S} \hat{U}
$$

Substituting the latter into Eq. A5, we obtain

$$
\left(\frac{d}{d t} \hat{U}^{\dagger}-\frac{i}{\hbar} \hat{U}^{\dagger} \hat{H}_{e f f, S}^{\dagger}-\hat{F}^{\dagger}\right) \hat{g}_{S} \hat{U}+\hat{U}^{\dagger} \hat{g}_{S}\left(\frac{d}{d t} \hat{U}+\frac{i}{\hbar} \hat{H}_{e f f, S} \hat{U}-\hat{F}\right)=0
$$

For simplicity we will assume operator $\hat{H}_{e f f}$ to be constant with time, i.e. we won't differentiate between $\hat{H}_{e f f}$ and $\hat{H}_{e f f, S}$.

The last equation is satisfied for sure if

$$
\frac{d}{d t} \hat{U}=-\frac{i}{\hbar} \hat{H}_{e f f} \hat{U}+\hat{F}, \frac{d}{d t} \hat{U}^{\dagger}=\frac{i}{\hbar} \hat{U}^{\dagger} \hat{H}_{e f f}^{\dagger}+\hat{F}^{\dagger}
$$

Multiplying Eqs. (A8) by the initial state vector $\left|\Psi_{0}\right\rangle$ from the right and from the left, we obtain the stochastic equation for the state vector and its Hermitian conjugate:

$$
\begin{gathered}
\frac{d}{d t}|\Psi\rangle=-\frac{i}{\hbar} \hat{H}_{e f f}|\Psi\rangle-\frac{i}{\hbar}|R(t)\rangle \\
\frac{d}{d t}\langle\Psi|=\frac{i}{\hbar}\langle\Psi| \hat{H}_{e f f}^{\dagger}+\frac{i}{\hbar}\langle R(t)|
\end{gathered}
$$

Where we introduced the notations $i \hbar \hat{F}\left|\Psi_{0}\right\rangle \Longrightarrow|R(t)\rangle,-i \hbar\left\langle\Psi_{0}\right| \hat{F}^{\dagger} \Longrightarrow\langle R(t)|$. We will also need Eqs. A9 and A10 in a particular basis $|\alpha\rangle$ :

$$
\begin{gathered}
\frac{d}{d t} C_{\alpha}=-\frac{i}{\hbar} \sum_{\nu}\left(\hat{H}_{e f f}\right)_{\alpha \nu} C_{\nu}-\frac{i}{\hbar} R_{\alpha} \\
\frac{d}{d t} C_{\alpha}^{*}=\frac{i}{\hbar} \sum_{\nu} C_{\nu}^{*}\left(\hat{H}_{e f f}^{\dagger}\right)_{\nu \alpha}+\frac{i}{\hbar} R_{\alpha}^{*}
\end{gathered}
$$

where $R_{\alpha}=\langle\alpha \mid R\rangle,\left(\hat{H}_{e f f}\right)_{\alpha \beta}=\left\langle\alpha\left|\hat{H}_{e f f}\right| \beta\right\rangle$.

Applying the same procedure to the standard Heisenberg equation (1) we obtain that in Eqs. A9,, $\mathrm{A} 10): \hat{H}_{e f f} \equiv \hat{H}_{e f f}^{\dagger}=\hat{H}$ and $\langle R(t)| \equiv 0$, which corresponds to the standard Schrödinger equation and its Hermitian conjugate. 
Note that intermediate relations $\mathrm{A} 8$ for the evolution operator and in particular operator $\hat{F}$ should not depend on the choice of a particular physical observable $g$ in the original Heisenberg-Langevin equation (A1). We assume that the Langevin operators in the original equation do not contradict this physically reasonable requirement.

In general, statistical properties of noise that ensure certain physically meaningful requirements impose certain constraints on the noise source $|R\rangle$ which enters the right-hand side of the stochastic equation for the state vector. In particular, it is natural to require that the statistically averaged quantity $\overline{|R\rangle}=0$. We will also require that the noise source $|R\rangle$ has the correlation properties that preserve the norm of the state vector averaged over the reservoir statistics:

$$
\overline{\langle\Psi(t) \mid \Psi(t)\rangle}=1
$$

\section{Noise correlator}

The solution to Eqs. A9 and (A10) can be formally written as

$$
\begin{aligned}
& |\Psi\rangle=e^{-\frac{i}{\hbar} \hat{H}_{e f f} t}\left|\Psi_{0}\right\rangle-\frac{i}{\hbar} \int_{0}^{t} e^{\frac{i}{\hbar} \hat{H}_{e f f}(\tau-t)}|R(\tau)\rangle d \tau, \\
& \langle\Psi|=\left\langle\Psi_{0}\right| e^{\frac{i}{\hbar} \hat{H}_{e f f}^{\dagger} t}+\frac{i}{\hbar} \int_{0}^{t}\langle R(\tau)| e^{-\frac{i}{\hbar} \hat{H}_{e f f}^{\dagger}(\tau-t)} d \tau,
\end{aligned}
$$

In the basis $|\alpha\rangle$, Eqs. A14, A15 can be transformed into

$$
\begin{aligned}
& C_{\alpha}=\left\langle\alpha\left|e^{-\frac{i}{\hbar} \hat{H}_{e f f} t}\right| \Psi_{0}\right\rangle-\frac{i}{\hbar} \int_{0}^{t}\left\langle\alpha\left|e^{\frac{i}{\hbar} \hat{H}_{e f f}(\tau-t)}\right| R(\tau)\right\rangle d \tau, \\
& C_{\alpha}^{*}=\left\langle\Psi_{0}\left|e^{\frac{i}{\hbar} \hat{H}_{e f f}^{\dagger} t}\right| \alpha\right\rangle+\frac{i}{\hbar} \int_{0}^{t}\left\langle R(\tau)\left|e^{-\frac{i}{\hbar} \hat{H}_{e f f}^{\dagger}(\tau-t)}\right| \alpha\right\rangle d \tau .
\end{aligned}
$$

In order to calculate the observables, we need to know the expressions for the averaged dyadic combinations of the amplitudes. We can find them using Eqs. (A11) and A12):

$$
\begin{aligned}
\frac{d}{d t} \overline{C_{\alpha} C_{\beta}^{*}}= & -\frac{i}{\hbar} \sum_{\nu}\left(H_{\alpha \nu}^{(h)} \overline{C_{\nu} C_{\beta}^{*}}-\overline{C_{\alpha} C_{\nu}^{*}} H_{\nu \beta}^{(h)}\right)-\frac{i}{\hbar} \sum_{\nu}\left(H_{\alpha \nu}^{(a h)} \overline{C_{\nu} C_{\beta}^{*}}+\overline{C_{\alpha} C_{\nu}^{*}} H_{\nu \beta}^{(a h)}\right) \\
& +\left(-\frac{i}{\hbar} \overline{C_{\beta}^{*} R_{\alpha}}+\frac{i}{\hbar} \overline{R_{\beta}^{*} C_{\alpha}}\right),
\end{aligned}
$$

Where we separated the Hermitian and anti-Hermitian components of the effective Hamiltonian: $\left\langle\alpha\left|\hat{H}_{\text {eff }}\right| \beta\right\rangle=H_{\alpha \beta}^{(h)}+H_{\alpha \beta}^{(a h)}$. Substituting Eqs. A16 and A17 into the last term 
in Eq. A18), we obtain

$$
\begin{aligned}
-\frac{i}{\hbar} \overline{C_{\beta}^{*} R_{\alpha}}+\frac{i}{\hbar} \overline{C_{\alpha} R_{\beta}^{*}}= & \frac{1}{\hbar^{2}} \int_{-t}^{0} \overline{\left\langle R(t+\xi)\left|e^{-\frac{i}{\hbar} \hat{H}_{e f f}^{\dagger} \xi}\right| \beta\right\rangle\langle\alpha \mid R(t)\rangle} d \xi \\
& +\frac{1}{\hbar^{2}} \int_{-t}^{0} \overline{\langle R(t) \mid \beta\rangle\left\langle\alpha\left|e^{\frac{i}{\hbar} \hat{H}_{e f f} \xi}\right| R(t+\xi)\right\rangle} d \xi .
\end{aligned}
$$

To proceed further with analytical results, we need to evaluate these integrals. The simplest situation is when the noise source terms are delta-correlated in time (Markovian). In this case only the point $\xi=0$ contributes to the integrals. As a result, Eq. (A18)) is transformed to

$$
\frac{d}{d t} \overline{C_{\alpha} C_{\beta}^{*}}=-\frac{i}{\hbar} \sum_{\nu}\left(H_{\alpha \nu}^{(h)} \overline{C_{\nu} C_{\beta}^{*}}-\overline{C_{\alpha} C_{\nu}^{*}} H_{\nu \beta}^{(h)}\right)-\frac{i}{\hbar} \sum_{\nu}\left(H_{\alpha \nu}^{(a h)} \overline{C_{\nu} C_{\beta}^{*}}+\overline{C_{\alpha} C_{\nu}^{*}} H_{\nu \beta}^{(a h)}\right)+D_{\alpha \beta},
$$

Where the correlator $D_{\alpha \beta}$ is defined by

$$
\overline{R_{\beta}^{*}(t+\xi) R_{\alpha}(t)}=\overline{R_{\beta}^{*}(t) R_{\alpha}(t+\xi)}=\hbar^{2} \delta(\xi) D_{\alpha \beta}
$$

The time derivative of the norm of the state vector is given by

$$
\frac{d}{d t} \sum_{\alpha} \overline{\left|C_{\alpha}\right|^{2}}=-\sum_{\alpha}\left[\frac{i}{\hbar} \sum_{\nu}\left(H_{\alpha \nu}^{(a h)} \overline{C_{\nu} C_{\alpha}^{*}}+\overline{C_{\alpha} C_{\nu}^{*}} H_{\nu \alpha}^{(a h)}\right)-D_{\alpha \alpha}\right]
$$

Clearly, the components $D_{\alpha \alpha}$ of the noise correlator need to compensate the decrease in the norm due to the anti-Hermitian component of the effective Hamiltonian. Therefore the expressions for $H_{\alpha \beta}^{(a h)}$ and $D_{\alpha \alpha}$ have to be mutually consistent. This is the manifestation of the fluctuation-dissipation theorem [31].

Note that the noise correlator could depend on the averaged combinations (e.g. dyadics) of the components of the state vector. This is because the noise source term $|R\rangle$ introduced above depends on the initial state $\left|\Psi_{0}\right\rangle$ and the evolution operator $\hat{U}$, and these quantities form the state vector components at any given time. Of course, what we call a "state vector" is the solution of the stochastic equation of motion, which is very different from the solution of the conventional Schrödinger equation for a closed system. In particular, we postulated the existence of the evolution operator $\hat{U}$ determined not only by the parameters of the dynamical system but also the properties of a dissipative reservoir, although we did not specify any particular expression for $\hat{U}$.

As an example, consider a simple diagonal anti-Hermitian operator $H_{\alpha \nu}^{(a h)}$ : 


$$
H_{\alpha \nu}^{(a h)}=-i \hbar \gamma_{\alpha} \delta_{\alpha \nu}
$$

And introduce the following models:

(i) Populations relax much slower than coherences (expected for condensed matter sys-

tems). In this case we can choose $D_{\alpha \neq \beta}=0, D_{\alpha \alpha}=2 \gamma_{\alpha} \overline{\left|C_{\alpha}\right|^{2}}$; within this model the population at each state will be preserved.

(ii) The state $\alpha=\alpha_{\text {down }}$ has a minimal energy, while the reservoir temperature $T=0$. In this case it is expected that all populations approach zero in equilibrium whereas the occupation number of the ground state approaches 1 , similar to the Weisskopf-Wigner model. The adequate choice of correlators is $D_{\alpha \neq \beta}=0, D_{\alpha \alpha} \propto \delta_{\alpha \alpha_{\text {down }}}, \gamma_{\alpha_{\text {down }}}=0$. The expression for the remaining nonzero correlator,

$$
D_{\alpha_{\text {down }} \alpha_{\text {down }}}=\sum_{\alpha \neq \alpha_{\text {down }}} 2 \gamma_{\alpha} \overline{\left|C_{\alpha}\right|^{2}}
$$

Ensures the conservation of the norm:

$$
\frac{d}{d t} \sum_{\alpha \neq \alpha_{\text {down }}} \overline{\left|C_{\alpha}\right|^{2}}=-\sum_{\alpha \neq \alpha_{\text {down }}} 2 \gamma_{\alpha} \overline{\left|C_{\alpha}\right|^{2}}=-\frac{d}{d t} \overline{\left|C_{\alpha_{\text {down }}}\right|^{2}} .
$$

This is an example of the correlator's dependence on the state vector that we discussed before.

\section{Comparison with the Lindblad method}

One can choose the anti-Hermitian Hamiltonian $H_{\alpha \beta}^{(a h)}$ and correlators $D_{\alpha \beta}$ in the stochastic equation of motion in such a way that Eq. $\mathrm{A} 19$ for the dyadics $\overline{C_{n} C_{m}^{*}}$ correspond exactly to the equations for the density matrix elements in the Lindblad approach. Indeed, the Lindblad form of the master equation has the form [23, 25]

$$
\frac{d}{d t} \hat{\rho}=-\frac{i}{\hbar}[\hat{H}, \hat{\rho}]+\hat{L}(\hat{\rho})
$$

where $\hat{L}(\hat{\rho})$ is the Lindbladian:

$$
\hat{L}(\hat{\rho})=-\frac{1}{2} \sum_{k} \gamma_{k}\left(\hat{l}_{k}^{\dagger} \hat{l}_{k} \hat{\rho}+\hat{\rho} \hat{l}_{k}^{\dagger} \hat{l}_{k}-2 \hat{l}_{k} \hat{\rho} \hat{l}_{k}^{\dagger}\right)
$$

Operators $\hat{l}_{k}$ in Eq. A25) and their number are determined by the model which describes the coupling of the dynamical system to the reservoir. The form of the relaxation operator given 
by Eq. A25 preserves automatically the conservation of the trace of the density matrix, whereas the specific choice of relaxation constants ensures that the system approaches a proper steady state given by thermal equilibrium or supported by an incoherent pumping.

Eq. A24 is convenient to represent in a slightly different form:

$$
\frac{d}{d t} \hat{\rho}=-\frac{i}{\hbar}\left(\hat{H}_{e f f} \hat{\rho}-\hat{\rho} \hat{H}_{e f f}^{\dagger}\right)+\delta \hat{L}(\hat{\rho})
$$

where

$$
\hat{H}_{e f f}=\hat{H}-i \hbar \sum_{k} \gamma_{k} \hat{l}_{k}^{\dagger} \hat{l}_{k}, \quad \delta \hat{L}(\hat{\rho})=\sum_{k} \gamma_{k} \hat{l}_{k} \hat{\rho} \hat{l}_{k}^{\dagger}
$$

Writing the anti-Hermitian component of the Hamiltonian in Eqs. (A11), A12) as

$$
H_{\alpha \beta}^{(a h)}=-i \hbar\left\langle\alpha\left|\sum_{k} \gamma_{k} \hat{l}_{k}^{\dagger} \hat{l}_{k}\right| \beta\right\rangle
$$

and defining the corresponding correlator of the noise source as

$$
\overline{R_{\beta}^{*}(t+\xi) R_{\alpha}(t)}=\hbar^{2} \delta(\xi) D_{\alpha \beta}, \quad D_{\alpha \beta}=\langle\alpha|\delta \hat{L}(\hat{\rho})| \beta\rangle_{\rho_{m n}=\overline{C_{n} C_{m}^{*}}}
$$

We obtain the solution in which averaged over noise statistics dyadics $\overline{C_{n} C_{m}^{*}}$ correspond exactly to the elements of the density matrix within the Lindblad method.

Instead of deriving the stochastic equation of evolution of the state vector from the Heisenberg-Langevin equations we could postulate it from the very beginning. After that, we could justify the choice of the effective Hamiltonian and noise correlators by ensuring that they lead to the same observables as the solution of the density matrix equations with the relaxation operator in Lindblad form [25, 33]. However, the demonstration of direct connection between the stochastic equation of evolution of the state vector and the Heisenberg-Langevin equation provides an important physical insight.

\section{Relaxation rates for coupled subystems interacting with a reservoir}

Whenever we have several coupled subsystems (such as electrons, photon modes, and phonons in this paper), each coupled to its reservoir, the determination of relaxation rates of the whole system becomes nontrivial. The problem can be solved if we assume that these "partial" reservoirs are statistically independent.In this case it is possible to add up partial Lindbladians and obtain the total effective Hamiltonian. 
Consider the Hamiltonian (11) of the system formed by a two-level electron system coupled to an EM mode field and dressed by a phonon field:

$$
\hat{H}=\hat{H}_{e m}+\hat{H}_{a}+\hat{H}_{p}+\hat{V}
$$

Here $\hat{H}_{e m}=\frac{\hbar \omega}{2}\left(\hat{c}^{\dagger} \hat{c}+\hat{c} \hat{c}^{\dagger}\right)$ is the Hamiltonian for a single EM mode field, $\hat{H}_{a}=W_{1} \hat{\sigma}^{\dagger} \hat{\sigma}+$ $W_{0} \hat{\sigma} \hat{\sigma}^{\dagger}$ is the Hamiltonian for a two-level "atom" with energy levels $W_{0,1}, \hat{H}_{p}=\frac{\hbar \Omega}{2}\left(\hat{b}^{\dagger} \hat{b}+\hat{b} \hat{b}^{\dagger}\right)$ is the Hamiltonian for a phonon mode, $\hat{V}=\hat{V}_{1}+\hat{V}_{2}$ the interaction Hamiltonian, where $\hat{V}_{1,2}$ describe the atom-photon and atom-photon-phonon coupling, respectively:

$$
\begin{gathered}
\hat{V}_{1}=-\left(\chi \hat{\sigma}^{\dagger} \hat{c}+\chi^{*} \hat{\sigma} \hat{c}^{\dagger}+\chi \hat{\sigma} \hat{c}+\chi^{*} \hat{\sigma}^{\dagger} \hat{c}^{\dagger}\right) \\
\hat{V}_{2}=-\left(\eta_{1} \hat{\sigma}^{\dagger} \hat{c} \hat{b}+\eta_{1}^{*} \hat{\sigma} \hat{c}^{\dagger} \hat{b}^{\dagger}+\eta_{2} \hat{\sigma}^{\dagger} \hat{c} \hat{b}^{\dagger}+\eta_{2}^{*} \hat{\sigma} \hat{c}^{\dagger} \hat{b}+\eta_{1} \hat{\sigma} \hat{c} \hat{b}+\eta_{1}^{*} \hat{\sigma}^{\dagger} \hat{c}^{\dagger} \hat{b}^{\dagger}+\eta_{2} \hat{\sigma} \hat{c} \hat{b}^{\dagger}+\eta_{2}^{*} \hat{\sigma}^{\dagger} \hat{c}^{\dagger} \hat{b}\right)
\end{gathered}
$$

where $\chi, \eta_{1}, \eta_{2}$ are coupling constants defined before.

Summing up the known (see e.g. [23, 25]) partial Lindbladians of two bosonic (infinite amount of energy levels) and one fermionic (two-level) subsystems, we obtain

$$
\begin{aligned}
L(\hat{\rho})= & -\frac{\gamma}{2} N_{1}^{T_{a}}\left(\hat{\sigma} \hat{\sigma}^{\dagger} \hat{\rho}+\hat{\rho} \hat{\sigma} \hat{\sigma}^{\dagger}-2 \hat{\sigma}^{\dagger} \hat{\rho} \hat{\sigma}\right)-\frac{\gamma}{2} N_{0}^{T_{a}}\left(\hat{\sigma}^{\dagger} \hat{\sigma} \hat{\rho}+\hat{\rho} \hat{\sigma}^{\dagger} \hat{\sigma}-2 \hat{\sigma} \hat{\rho} \hat{\sigma}^{\dagger}\right) \\
& -\frac{\mu_{\omega}}{2} \bar{n}_{\omega}^{T_{e m}}\left(\hat{c} \hat{c}^{\dagger} \hat{\rho}+\hat{\rho} \hat{c}^{\dagger} \hat{c}-2 \hat{c}^{\dagger} \hat{\rho} \hat{c}\right)-\frac{\mu_{\omega}}{2}\left(\bar{n}_{\omega}^{T_{e m}}+1\right)\left(\hat{c}^{\dagger} \hat{c} \hat{\rho}+\hat{\rho} \hat{c} \hat{c}^{\dagger}-2 \hat{c} \hat{\rho} \hat{c}^{\dagger}\right) \\
& -\frac{\mu_{\Omega}}{2} \bar{n}_{\Omega}^{T_{p}}\left(\hat{b} \hat{b}^{\dagger} \hat{\rho}+\hat{\rho} \hat{b}^{\dagger} \hat{b}-2 \hat{b}^{\dagger} \hat{\rho} \hat{b}\right)-\frac{\mu_{\Omega}}{2}\left(\bar{n}_{\Omega}^{T_{p}}+1\right)\left(\hat{b} \hat{b}^{\dagger} \hat{\rho}+\hat{\rho} \hat{b} \hat{b}^{\dagger}-2 \hat{b} \hat{\rho} \hat{b}^{\dagger}\right)
\end{aligned}
$$

where $\gamma, \mu_{\omega}$ and $\mu_{\Omega}$ are partial relaxation rates of the subsystems,

$$
N_{0,1}^{T_{a}}=\left(1+e^{-\frac{W_{1}-W_{0}}{T_{a}}}\right)^{-1} e^{-\frac{W_{0,1}-W_{0}}{T_{a}}}, \bar{n}_{\omega}^{T_{e m}}=\left(e^{\frac{\hbar \omega}{T_{e m}}}-1\right)^{-1}, \bar{n}_{\Omega}^{T_{p}}=\left(e^{\frac{\hbar \Omega}{T p}}-1\right)^{-1},
$$

$T_{a, e m, p}$ are the temperatures of partial reservoirs. For the Lindblad master equation in the form Eq. (A26) we get

$$
\hat{H}_{e f f}=\hat{H}-i \hat{\Gamma}
$$

where

$\hat{\Gamma}=\frac{\hbar}{2}\left\{\gamma\left(N_{1}^{T_{a}} \hat{\sigma} \hat{\sigma}^{\dagger}+N_{0}^{T_{a}} \hat{\sigma}^{\dagger} \hat{\sigma}\right)+\mu_{\omega}\left[\bar{n}_{\omega}^{T_{e m}} \hat{c} \hat{c}^{\dagger}+\left(\bar{n}_{\omega}^{T_{e m}}+1\right) \hat{c}^{\dagger} \hat{c}\right]+\mu_{\Omega}\left[\bar{n}_{\Omega}^{T_{p}} \hat{b} \hat{b}^{\dagger}+\left(\bar{n}_{\Omega}^{T_{p}}+1\right) \hat{b}^{\dagger} \hat{b}\right]\right\}$.

Using the effective Hamiltonian given by Eqs. A32, A33), we arrive at the stochastic equation for the state vector in the following form:

$$
\frac{d}{d t} C_{\alpha n 0}=-i \frac{W_{0}+\hbar \omega\left(n+\frac{1}{2}\right)+\hbar \Omega\left(\alpha+\frac{1}{2}\right)}{\hbar} C_{\alpha n 0}-\frac{i}{\hbar}\langle\alpha|\langle n|\langle 0|\hat{V}| \Psi\rangle-\gamma_{\alpha n 0} C_{\alpha n 0}-\frac{i}{\hbar} R_{\alpha n 0},
$$


$\frac{d}{d t} C_{\alpha n 1}=-i \frac{W_{1}+\hbar \omega\left(n+\frac{1}{2}\right)+\hbar \Omega\left(\alpha+\frac{1}{2}\right)}{\hbar} C_{\alpha n 1}-\frac{i}{\hbar}\langle\alpha|\langle n|\langle 1|\hat{V}| \Psi\rangle-\gamma_{\alpha n 1} C_{\alpha n 1}-\frac{i}{\hbar} R_{\alpha n 1}$

where

$$
\begin{aligned}
& \gamma_{\alpha n 0}=\frac{\gamma}{2} N_{1}^{T_{a}}+\frac{\mu_{\omega}}{2}\left[\bar{n}_{\omega}^{T_{e m}}(n+1)+\left(\bar{n}_{\omega}^{T_{e m}}+1\right) n\right]+\frac{\mu_{\Omega}}{2}\left[\bar{n}_{\Omega}^{T_{p}}(\alpha+1)+\left(\bar{n}_{\Omega}^{T_{p}}+1\right) \alpha\right], \\
& \gamma_{\alpha n 1}=\frac{\gamma}{2} N_{0}^{T_{a}}+\frac{\mu_{\omega}}{2}\left[\bar{n}_{\omega}^{T_{e m}}(n+1)+\left(\bar{n}_{\omega}^{T_{e m}}+1\right) n\right]+\frac{\mu_{\Omega}}{2}\left[\bar{n}_{\Omega}^{T_{p}}(\alpha+1)+\left(\bar{n}_{\Omega}^{T_{p}}+1\right) \alpha\right],
\end{aligned}
$$

Eqs. A36, A37 determine the rules of combining the "partial" relaxation rates for several coupled subsystems.

[1] M. Aspelmeyer, T. J. Kippenberg, and F. Marquardt, Rev. Mod. Phys. 86, 1391 (2014).

[2] P. Meystre, Ann. Phys. 525, 215 (2013).

[3] J.-M. Pirkkalainen, S.U. Cho, F. Massel, J. Tuorila, T.T. Heikkila, P.J. Hakonen, and M.A. Sillanpaa, Nat. Comm. 6, 6981 (2015).

[4] Y. Chu, P. Kharel, W. H. Renninger, L. D. Burkhart, L.Frunzio, P. T. Rakich, R. J. Schoelkopf, Science 358, 199 (2017).

[5] S, Hong, R. Riedinger, I. Marinkovic, et al., Science 358, 203 (2017).

[6] P. Arrangoiz-Arriola, E. A. Wollack, Z. Wang, M. Pechal, W. Jiang, T. P. McKenna, J. D. Witmer, R. Van Laer, and A.H. Safavi-Naeini, Nature 571, 537 (2019).

[7] F. Benz, M. K. Schmidt, A. Dreismann, R. Chikkaraddy, Y. Zhang, A. Demetriadou, C. Carnegie, H. Ohadi, B. de Nijs, R. Esteban, J. Aizpurua, and J. J. Baumberg, Science 354, 726 (2016).

[8] K.-D. Park, E. A. Muller, V. Kravtsov, P. M. Sass, J. Dreyer, J. M. Atkin, and M. B. Raschke, Nano Lett. 16, 479 (2016).

[9] M. S. Tame, K. R. McEnery, S. K. Ozdemir, J. Lee, S. A. Maier, and M. S. Kim, Nat. Phys. 9, 329 (2013).

[10] F.C.B. Maia, B.T. OCallahan, A.R. Cadore, I.D. Barcelos, L.C. Campos, K. Watanabe, T. Taniguchi, C. Deneke, A. Belyanin, M.B. Raschke, and R.O. Freitas, Nano Lett. 19, 708 (2019). 
[11] R. Chikkaraddy, B. de Nijs, F. Benz, S. J. Barrow, O. A. Scherman, E. Rosta, A. Demetriadou, P. Fox, O. Hess, and J. J. Baumberg, Nat. 535, 127 (2016).

[12] A. Sipahigil, R. E. Evans, D. D. Sukachev, et al., Science 354, 847 (2016).

[13] T. Yoshie, A. Scherer, J. Hendrickson, G. Khitrova, H. M. Gibbs, G. Rupper, C. Ell, O. B. Shchekin, and D. G. Deppe, Nature 432, 200 (2004).

[14] J. P. Reithmaier, G. Sek A. Loffler, C. Hofmann, S. Kuhn, S. Reitzenstein, L. V. Keldysh, V. D. Kulakovskii, T. L. Reinecke, and A. Forchel, Nature 432, 197 (2004).

[15] H. Leng, B. Szychowski, M.-C. Daniel, and M. Pelton, Nat Commun. 9, 4012 (2018).

[16] O. Bitton, S. N. Gupta, and G. Haran, Nanophotonics 8, 559 (2019).

[17] K.-D. Park, M. A. May, H. Leng, J. Wang, J. A. Kropp, T. Gougousi, M. Pelton, M. B. Raschke, Sci. Adv. 2019;5: eaav5931.

[18] K. J. Satzinger, Y. P. Zhong, H. Chang, et al., Nature 563, 661665 (2018).

[19] A. Bienfait, Y. P. Zhong, H.-S. Chang, M.-H. Chou, C. R. Conner, E. Dumur, J. Grebel, G. A. Peairs, R. G. Povey, K. J. Satzinger, and A. N. Cleland, Phys. Rev. X 10, 021055 (2020).

[20] U. Delic et al., Science 367, 892 (2020).

[21] N. Bloembergen, Nonlinear Optics (World Scientific, 1996).

[22] Y. A. Bogoliubov and N. N. Mitropolsky, Asymptotic Methods in the Theory of Nonlinear Oscillations (Gordon and Breach, 1961).

[23] M. O. Scully and M. S. Zubairy, Quantum Optics (Cambridge University Press, Cambridge, 1997)

[24] P. Forn-Diaz, L. Lamata, E. Rico, J. Kono, and E. Solano, Rev. Mod. Phys. 91, 025005 (2019).

[25] M. B. Plenio and P. L. Knight, Rev. Mod. Phys. 70, 101 (1998).

[26] M.Tokman, Y. Wang, I. Oladyshkin, A. R. Kutayiah, and A. Belyanin, Phys. Rev. B 93, $235422(2016)$.

[27] C. Gardiner and P. Zoller, Quantum Noise (Springer-Verlag, Berlin, Heidelberg, 2004).

[28] M. Tokman, X. Yao, and A. Belyanin, Phys. Rev. Lett. 110, 077404 (2013).

[29] M. Erukhimova and M. Tokman, Phys. Rev A 95, 013807 (2017).

[30] M. Tokman, Z. Long, S. Almutairi, Y. Wang, V.Vdovin, M. Belkin, and A. Belyanin, APL Photonics 4, 034403 (2019).

[31] L.D. Landau, E.M. Lifshitz, Statistical Physics, Part 1 (Pergamon, Oxford, 1965).

[32] K. H. Madsen and P. Lodahl, New J. of Phys. 15, 025013 (2013). 
[33] K. Blum, Density Matrix Theory and Applications (Springer, Heidelberg, 2012). 